Florida International University

FIU Digital Commons

7-19-2018

\title{
Characterization of the Uranium-Bearing Phases Produced by Novel Remediation Technologies for Sequestration of Mobile Radiological Contaminants in the Hanford 200 Area Vadose Zone
}

Robert Michael Lapierre

Florida International University, rlapi001@fiu.edu

Follow this and additional works at: https://digitalcommons.fiu.edu/etd

Part of the Analytical Chemistry Commons, Earth Sciences Commons, and the Environmental Chemistry Commons

\section{Recommended Citation}

Lapierre, Robert Michael, "Characterization of the Uranium-Bearing Phases Produced by Novel Remediation Technologies for Sequestration of Mobile Radiological Contaminants in the Hanford 200 Area Vadose Zone" (2018). FIU Electronic Theses and Dissertations. 3847.

https://digitalcommons.fiu.edu/etd/3847

This work is brought to you for free and open access by the University Graduate School at FIU Digital Commons. It has been accepted for inclusion in FIU Electronic Theses and Dissertations by an authorized administrator of FIU Digital Commons. For more information, please contact dcc@fiu.edu. 


\title{
FLORIDA INTERNATIONAL UNIVERSITY
}

Miami, Florida

CHARACTERIZATION OF THE URANIUM-BEARING PHASES PRODUCED BY NOVEL REMEDIATION TECHNOLOGIES FOR SEQUESTRATION OF MOBILE RADIOLOGICAL CONTAMINANTS IN THE HANFORD 200 AREA VADOSE ZONE

\author{
A thesis submitted in partial fulfillment of \\ the requirements for the degree of \\ MASTER OF SCIENCE \\ in \\ CHEMISTRY \\ by
}

Robert Michael Lapierre 
To: Dean Michael R. Heithaus

College of Arts, Sciences and Education

This thesis, written by Robert Michael Lapierre, and entitled Characterization of the Uranium-Bearing Phases Produced by Novel Remediation Technologies for Sequestration of Mobile Radiological Contaminants in the Hanford 200 Area Vadose Zone, having been approved in respect to style and intellectual content, is referred to you for judgment.

We have read this thesis and recommend that it be approved.

$\begin{array}{r}\text { Yelena Katsenovich } \\ \hline \text { Yi Xiao } \\ \hline \text { Yong Cai, Major Professor }\end{array}$

Date of Defense: July 19, 2018

The thesis of Robert Michael Lapierre is approved.

Dean Michael R. Heithaus

College of Arts, Sciences and Education

Andrés G. Gil

Vice President for Research and Economic Development and Dean of the University Graduate School

Florida International University, 2018 
(C) Copyright 2018 by Robert Michael Lapierre

All rights reserved. 


\section{DEDICATION}

This work is dedicated to my family, who always made me believe that there was more than I could see. To my mentor, advisors, and friends with the DOE-FIU Science and Technology Workforce Development Program. And to Ashley, whose encouragement, support, and commitment held me up and kept me going.

If I am the sum of all my experiences, then nothing I've done has been a waste of time. Journey before destination. 


\section{ACKNOWLEDGMENTS}

I would like to acknowledge and thank my committee for their guidance; Dr. Yong Cai, Dr. Yi Xiao, and Dr. Yelena Katsenovich. I want to express my gratitude to Dr. Dawn Wellman and Dr. Jim Szecsody with Pacific Northwest National Laboratory (PNNL) for their support and mentorship during my internships and beyond. I'd also like to thank Tom Beasley with FIU's FCAEM who taught me so much about electron microscopy. I would like to thank Dr. Leonel Lagos, the Department of Energy Office of Environmental Management, and the DOE-FIU Science and Technology Workforce Development Program who helped make this possible. Also, a very special thanks to Dr. Ravi Gudavalli, Dr. Hilary Emerson, and my fellow DOE Fellows who always made themselves available. 
ABSTRACT OF THE THESIS

CHARACTERIZATION OF THE URANIUM-BEARING PHASES PRODUCED BY NOVEL REMEDIATION TECHNOLOGIES FOR SEQUESTRATION OF MOBILE RADIOLOGICAL CONTAMINANTS IN THE HANFORD 200 AREA VADOSE ZONE by

Robert Michael Lapierre

Florida International University, 2018

Miami, Florida

Professor Yong Cai, Major Professor

Of the many toxic chemicals released into the Hanford vadose zone over the decades of nuclear weapons production, uranium has emerged as a contaminant of significant interest. The ammonia gas injection remediation method has been identified as a promising approach towards mitigating the risks to the ecosystem by limiting the mobility of the radionuclide in the vadose zone. The remediation method was replicated using synthetic porewater solutions with a range of constituent concentrations equal to that of the Hanford 200 Area vadose zone. The uranium-bearing products of the remediation method were characterized using kinetic phosphorescence analysis for aqueous uranium, scanning electron microscopy with energy dispersive spectroscopy and electron microprobe for imaging and elemental analysis, and a sequential extraction procedure modified for the sample precipitates. Evaluation revealed that the resultant uranium-bearing solids likely took the form of uranium-silicates and uranium carbonates, with the latter being precipitated primarily in mid-to-high bicarbonate samples. 


\section{TABLE OF CONTENTS}

\section{CHAPTER}

PAGE

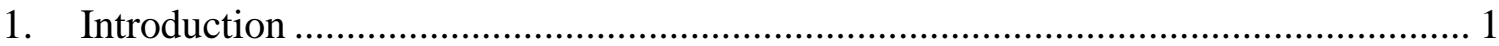

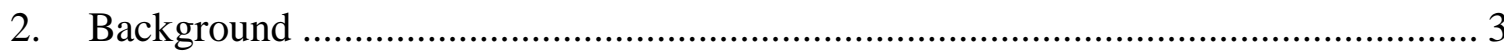

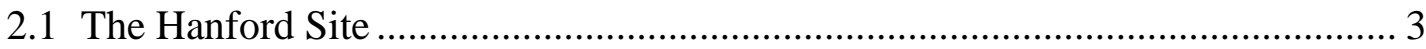

2.2 The $\mathrm{NH}_{3}$ Gas Remediation Strategy....................................................... 5

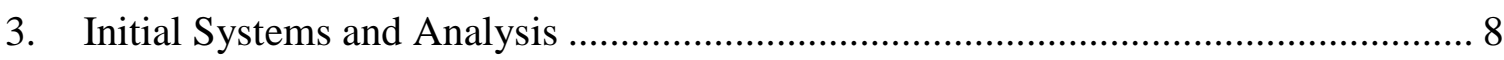

3.1 Sample Preparation Methods ........................................................................... 8

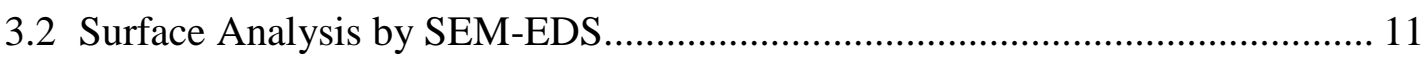

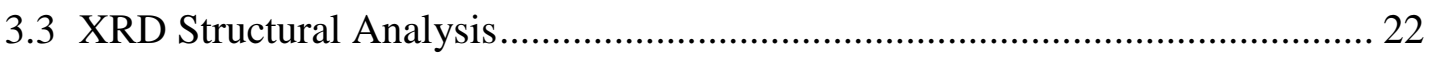

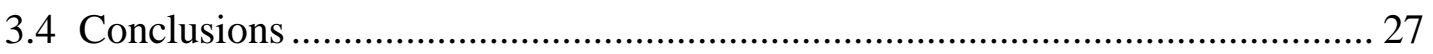

4. Optimization and Analysis of the Products of the $\mathrm{NH}_{3}$ Injection Method............... 29

4.1 Synthetic Pore Water System Optimization..................................................... 29

4.2 Characterization of the Uranium-Bearing Solid Phase .................................... 35

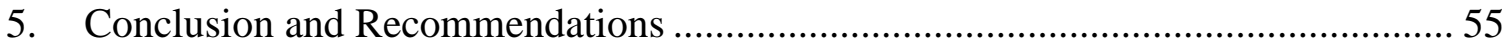

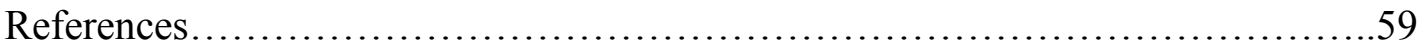




\section{LIST OF TABLES}

TABLE

PAGE

Table 1. Stock Solutions and Sample Mixtures ........................................................... 9

Table 2. Sample Labels with Development Time....................................................... 11

Table 3. Condensed Series of Samples with Development Time ................................... 19

Table 4. Stock Solution \& Synthetic Pore Water Concentrations for Sample Preparation

30

Table 5. Synthetic Pore Water Solution Concentrations \& Labels................................. 32

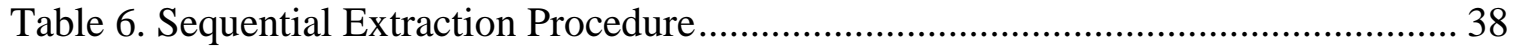

Table 7. Extraction Solution Volumes - by Sample ................................................. 38 


\section{LIST OF FIGURES}

FIGURE

PAGE

Figure 1. Precipitates loaded on studs using carbon tape and mounted for SEM. 12

Figure 2. SEM image and EDS data for a $3 \mathrm{mM}$ bicarbonate, $0 \mathrm{mM}$ calcium sample..... 15

Figure 3. SEM image and EDS data for a $3 \mathrm{mM}$ bicarbonate, $5 \mathrm{mM}$ calcium sample..... 15

Figure 4. SEM image and EDS data for a $50 \mathrm{mM}$ bicarbonate, $0 \mathrm{mM}$ calcium sample... 17

Figure 5. SEM image and EDS data for a $50 \mathrm{mM}$ bicarbonate, $5 \mathrm{mM}$ calcium sample... 17

Figure 6. Timeline of uranium-rich region change with increasing development time

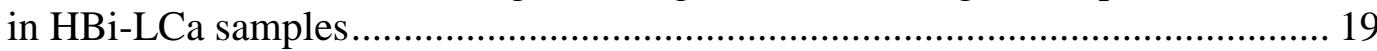

Figure 7. Timeline of uranium-rich region change with increasing development time in HBi-HCa samples ................................................................................... 19

Figure 8. SEM image and EDS data for the $50 \mathrm{mM}$ bicarbonate, $5 \mathrm{mM}$ calcium sample with a 1-month development time

Figure 9. Comparison of the diffraction patterns for the calcium-free (left) and $5 \mathrm{mM}$ calcium-containing (right) precipitate samples with the pattern for nitratine $\left(\mathrm{NaNO}_{3}\right)$

Figure 10. Comparison of the diffraction patterns of calcium-free (left) and $5 \mathrm{mM}$ calcium-containing (right) precipitate samples with the pattern for Cejkaite $\left(\mathrm{Na}_{4}\left(\mathrm{UO}_{2}\right)\left(\mathrm{CO}_{3}\right)_{3}\right)$ 25

Figure 11. SEM image and EDS data for the ground HBi-LCa-2W sample .................. 26

Figure 12. SEM image and EDS data showing a uranium rich region in the ground $\mathrm{HBi}-\mathrm{HCa}-2 \mathrm{~W}$ sample.

Figure 13. SEM image and EDS data showing fragmented remains of needle-like structures in the ground $\mathrm{HBi}-\mathrm{HCa}-2 \mathrm{~W}$ sample

Figure 14. $\mathrm{NH}_{3}$ injection of the low, mid, and high bicarbonate synthetic pore water base solutions.

Figure 15. Response surface diagrams displaying filtrate solution uranium retention for the original (Group A) and duplicate/rinsed (Group B) samples.... 33

Figure 16. Epoxy molds before (a) and after (b) filling with resin + sample mixtures. ... 40 
Figure 17. SEM images and EDS data for unrinsed low bicarbonate $(5 \mathrm{mM})$ samples ... 42

Figure 18. SEM images and EDS data for rinsed low bicarbonate $(5 \mathrm{mM})$ samples 43

Figure 19. SEM image and EDS data for point analysis of specimens from the rinsed, $25 \mathrm{mM}$ bicarbonate, zero calcium precipitate (left) and the unrinsed, $25 \mathrm{mM}$ bicarbonate, $5 \mathrm{mM}$ calcium precipitate (right)

Figure 20. SEM image and EDS data for point analysis of specimens from the unrinsed, $50 \mathrm{mM}$ bicarbonate, zero calcium precipitate (left) and the unrinsed, $50 \mathrm{mM}$ bicarbonate, $5 \mathrm{mM}$ calcium precipitate (right).

Figure 21. Sequential uranium extraction of sample precipitates (including rinse).

Figure 22. Uranium Extraction Distribution for Unrinsed (Group A) Samples. 48

Figure 23. Uranium Extraction Distribution for Rinsed (Group B) Samples. 48

Figure 24. EPMA micrograph and corresponding elemental maps for the unrinsed, low bicarbonate $(5 \mathrm{mM})$, low calcium $(0 \mathrm{mM})$ sample

Figure 25. EPMA micrograph and corresponding elemental maps for the unrinsed, low bicarbonate $(5 \mathrm{mM}), 10 \mathrm{mM}$ calcium sample

Figure 26. EPMA micrograph and corresponding elemental maps for the unrinsed, high bicarbonate $(50 \mathrm{mM})$, low calcium $(0 \mathrm{mM})$ sample 53

Figure 27. EPMA micrograph and corresponding elemental maps for the unrinsed, high bicarbonate $(50 \mathrm{mM}), 10 \mathrm{mM}$ calcium sample 


\section{ABBREVIATIONS AND ACRONYMS}

$\begin{array}{ll}\text { AT\% } & \text { Atomic Percentage } \\ \text { CERCLA } & \begin{array}{l}\text { Comprehensive Environmental Response, Compensation, and Liability } \\ \text { Act }\end{array} \\ \text { DIW } & \text { Deionized Water } \\ \text { DST } & \text { Double-Shell Tank } \\ \text { EDS } & \text { Energy Dispersive Spectroscopy } \\ \text { EMSL } & \text { Environmental Molecular Sciences Laboratory } \\ \text { EPMA } & \text { Electron Probe Microanalyzer } \\ \text { FCAEM } & \text { Florida Center for Analytical Electron Microscopy } \\ \text { KPA } & \text { Kinetic Phosphorescence Analyzer } \\ \text { PNNL } & \text { Pacific Northwest National Laboratory } \\ \text { SEM } & \text { Scanning Electron Microscopy } \\ \text { SST } & \text { Single-Shell Tank } \\ \text { XRD } & \text { X-Ray Diffraction }\end{array}$




\section{Introduction}

Over 70 years ago, the Hanford Site served as a key nuclear production facility in the country's burgeoning nuclear weapons production complex. The site produced fuel for nuclear weapons for more than four decades before being decommissioned. In the process, Hanford would amass vast amounts of radiologically contaminated liquid and solid waste which would be managed primarily through treatment and underground storage. Today, Hanford is one of the world's most massive ongoing cleanup efforts (Gephart, 2003; Gerber, 1992; U.S. Department of Energy, 2018). The improper storage and disposal of the legacy waste from uranium extraction and plutonium production has resulted in the uncontrolled release of hazardous waste into the local environment. The uncontrolled release of these pollutants has resulted in radiological contamination of the vadose zone, the unsaturated region between the topsoil and the water table, threatening the Columbia River and surrounding ecosystem.

In response to the massive release of waste into the environment, the US Department of Energy launched what would grow to become a multi-billion-dollar remediation effort. The Hanford Site would be classified as a National Priority and Superfund site, designating it as one of the most critical cases of hazardous waste contamination posing a threat to human health and the environment. The remediation effort would focus on, among other things, the long-term storage of contaminated waste and the mitigation of soil and groundwater contamination (Hughes, Douglas, \& Marske, 1994; Liu et al., 2006; U.S. Department of Energy - Richland Operations Office, 2008). Among the tasks undertaken was the sequestration of mobile contaminants in the Hanford vadose zone. Uranium, a key 
radiological contaminant in the Hanford subsurface, poses a significant long-term threat to ecosystem as it drifts towards the nearby Columbia River. Of the proposed technologies for mitigating this risk, the in-situ $\mathrm{pH}$ manipulation using $\mathrm{NH}_{3}$ gas has shown promise warranting further investigation (Szecsody, Truex, Zhong, Williams et al., 2010).

In order to further the understanding of the ammonia gas injection remediation method for the Hanford vadose zone, continued investigation of the system, its products, and the role that pore water constituents play in sequestering uranium are required. The objective of my study is to replicate the proposed $\mathrm{NH}_{3}$ gas injection remediation technology on the bench scale using a synthetic porewater solution, prepared to replicate conditions relevant to the Hanford 200 Area vadose zone, and the characterization of the uranium-bearing products. The results of the study will directly supplement ongoing research regarding the stability of the uranium species produced under vadose zone conditions and thereby the efficacy of the subsurface remediation method for sites like the Hanford 200 Area. 


\section{Background}

\subsection{The Hanford Site}

The Hanford Site was commissioned by the United States government in the midst of World War II under a shroud of secrecy (Gerber, 1992). The now decommissioned facility was once a part of the nation's nuclear weapons complex and a key project site for the Manhattan Project. The reactors built there would go on to produce more than 50 metric tons of weapons grade plutonium (Gephart, 2003); including the plutonium that fueled one of the only two nuclear weapons every used in war. Among the areas that made up the site were the 100 Area, built alongside the Columbia river to house and support nuclear reactors, the 200 Area, in the Central Plateau region where fuel was reprocessed and waste was stored and disposed of, and the 300 Area where fuel was prepared (Gephart, 2003).

During the wartime effort, regretful decisions were made involving the handling of the of various types of solid and liquid hazardous waste produced in nuclear fuel cycle. These decisions include piping waste into underground tanks, burying solid waste in trenches, and releasing liquid waste to the soil and groundwater. This release of waste occurred primarily in the 200 Area where tank farms, cribs, and trenches were located. Over the 40+ years of operation, more than 400 billion gallons of contaminated liquid waste were either accidentally spilled or improperly disposed of to the soil and groundwater (Hartman \& Dresel, 1998). More than 200,000 kg of uranium, the principal component of the nuclear fuel cycle, is known to have been discharged into the subsurface (Zachara et al., 2007). More than 50 million gallons of liquid radioactive waste still remain in the 149 single-shell tanks (SSTs), which were originally designed for 20 year lifespans, and the 28 newer 
double-shell tanks (DSTs) (Gephart, 2003; Gephart, 2010; U.S. Department of Energy, 2018) buried on the Hanford Site. Over $40 \%$ of the SSTs have been confirmed to be leaking their radioactive contents into the surrounding soil (Gephart, 2003; Gephart, 2010).

The U.S. Department of Energy has taken momentous steps to protect the people and the environment from the anthropogenic pollutants that stand to affect the ecosystem surrounding the Hanford Site. Under the Comprehensive Environmental Response, Compensation, and Liability Act (CERCLA), also known as the Superfund, the site was classified as a National Priority, demanding long-term remediation planning to mitigate the looming environmental hazards. These remediation efforts include decommissioning and amendment projects that focus on addressing the long-term storage of contaminated waste and the mitigation of soil and groundwater contamination (Hughes et al., 1994; Liu et al., 2009; U.S. Department of Energy - Richland Operations Office, 2008). The Hanford vadose zone, in the 200 Area in particular, has become a matter of priority because of the hundreds of thousands of tons of toxic waste it holds (Gephart, 2003). Though characterizations have shown that a variety of contaminants reside inside of the vadose zone, uranium (U) has emerged as a contaminant of interest for researchers (Serne et al., 2008).

The vadose zone is the unsaturated region of soil that resides between the topsoil and the water table. The principle concern about its contamination is the mobilization of pollutants like uranium down through the vadose zone to the water table and into the Columbia River, threatening the surrounding ecosystem. In response to the challenge, extensive studies on the conditions of the Hanford vadose zone have been done throughout the years (Cantrell, 
Zachara, Dresel, Krupka, \& Serne, 2007; Gee, Oostrom, Freshley, Rockhold, \& Zachara, 2007; Hartman \& Dresel, 1998; Serne et al., 2008). The conditions are described to be oxidizing and mildly basic, with a typical $\mathrm{pH}$ range from $7.5-8.5$. Uranium is typically found in the hexavalent $\mathrm{U}(\mathrm{VI})$ oxidation state which tends to form more mobile soluble carbonate species (Zachara et al., 2007).

Researchers at Pacific Northwest National Laboratory (PNNL) have tested and proposed various remediation methods for mitigating the potential problems posed by the Hanford vadose zone contaminants (U.S. Department of Energy - Richland Operations Office, 2008). Aqueous remediating amendments for the Hanford vadose zone are typically viewed as nonstarters because of the desire to avoid adding in liquids to the subsurface which could spur further mobilization. Several gas-delivered solutions have been evaluated with varying degrees of success (Szecsody et al., 2010; U.S. Department of Energy Richland Operations Office, 2008). Of the proposed technologies for accomplishing this task, the in-situ $\mathrm{pH}$ manipulation using $\mathrm{NH}_{3}$ gas has shown significant promise, prompting further investigation (Szecsody et al., 2012; Zhong, Szecsody, Truex, Williams, \& Liu, 2015).

\subsection{The $\mathrm{NH}_{3}$ Gas Remediation Strategy}

The Department of Energy's Deep Vadose Zone Treatability Test Plan for the Hanford Central Plateau details candidate technologies proposed for the remediation of the Hanford Deep Vadose Zone (U.S. Department of Energy - Richland Operations Office, 2008). Published pilot studies investigating the proposed gas-transported reactants examined a series of gas-phase amendments for application (Szecsody et al., 2010). The study 
established the ammonia $\left(\mathrm{NH}_{3}\right)$ injection method as a potentially viable remediation solution for the sequestration of uranium in the subsurface. The assertion made was that the remediation would function by promoting the dissolution of solid phases followed by the re-precipitation solid species and coating of uranyl phases, limiting mobility (Szecsody et al., 2010).

The injection of $\mathrm{NH}_{3}$ gas into the subsurface functions to changes the chemistry of the unsaturated vadose zone as the gas diffuses through the soil and into the pore water. The pore water is the water residing in the interstitial space left by the grains of soil. As ammonia is dissolved into the aqueous pore water, ammonium $\left(\mathrm{NH}_{4}{ }^{+}\right)$is formed, and the subsurface $\mathrm{pH}$ increases from the natural range of 7-8 to 11 . Under these alkaline conditions, the partial dissolution of solid phase minerals (i.e.: aluminosilicates) present in the system is strongly promoted. The re-establishment of natural $\mathrm{pH}$ conditions can occur without amendment, likely through the natural buffer capacity of the soil, the diffusion of $\mathrm{CO}_{2}$ containing air, or the evaporation of aqueous ammonia. This re-establishment of $\mathrm{pH}$ promotes the re-precipitation of mineral solid phases, likely co-precipitating or coating uranium species (Szecsody et al., 2010; Szecsody et al., 2012; Zhong et al., 2015). Upon the re-establishment of natural $\mathrm{pH}$ conditions, the system re-precipitates the dissolved mineral phases, likely co-precipitating uranyl solids and forming a coating on said uranium species (Szecsody et al., 2010; Szecsody et al., 2012; Zhong et al., 2015). Contrary to the goal of limiting the contaminant mobility, these high $\mathrm{pH}$ conditions initially favor the dissolution and mobilization of solid and adsorbed uranyl phases present in the subsurface. Conveniently, this initial mobilization of uranyl phases is not a problem for the 200 Area vadose zone because of the low advection rates in this unsaturated region. 
Pacific Northwest National Laboratory scientists have published extensive studies of the ammonia gas remediation method for application to the Hanford vadose zone in both peerreviewed journals (Szecsody et al., 2010; Zhong et al., 2015) and internal reports (Szecsody, Truex, Zhong, Qafoku et al., 2010; Szecsody et al., 2012). These laboratory scale studies, performed using contaminated soil samples retrieved from the Hanford, have indicated that Na-boltwoodite $\left(\mathrm{Na}\left(\mathrm{UO}_{2}\right)\left(\mathrm{SiO}_{3} \mathrm{OH}\right) \cdot \mathrm{H}_{2} \mathrm{O}\right)$ would be the primary uranylphase present in the untreated vadose zone soil. Post-treatment soil showed reduced mobility of those uranyl phases through sequential extractions (Szecsody et al., 2010; Szecsody et al., 2010; Szecsody et al., 2012). Contrastingly, my study uses a synthetic pore water solution, without the presence of soil, to represent the Hanford 200 Area pore water as characterized by Serne et al (2008). 


\section{Initial Systems and Analysis}

The present study was designed to build on past research while simultaneously supporting the ongoing efforts surrounding the environmental remediation of the Hanford Site vadose zone. In particular, the prior research of scientists with Pacific Northwest National Laboratory (Szecsody et al., 2010; Szecsody et al., 2010) and their collaborators with Florida International University’s Applied Research Center (Lagos et al., 2012; Lagos et al., 2013; Lagos et al., 2014) was used to design a set of initial exploratory experiments which would serve as the investigation's launching point. These preliminary data, primarily the results of qualitative analysis, would be used determine how to best apply the ammonia gas remediation method on the laboratory scale. The observations would be used to shape the more comprehensive, quantitative experiments discussed in the subsequent Optimization and Analysis of the Products of the $\mathrm{NH}_{3}$ Injection Method section.

\subsection{Sample Preparation Methods}

The experimental approach used in my introductory investigation involved the preparation of multiple sets of synthetic pore water solutions designed to meet conditions relevant to the vadose zone Hanford Site 200 Area. The concentrations were determined using the range reported as found in the 200 Area vadose zone by Serne et al. (2008). For my study, sample solutions were limited to five key constituents ( $\mathrm{U}, \mathrm{Si}, \mathrm{Al}, \mathrm{HCO}_{3}{ }^{-}$, and $\mathrm{Ca}$ ) thought to be most relevant to the sequestration study. In all samples, the concentrations of uranium (0.84 mM/200 ppm), silica $(100 \mathrm{mM})$, and aluminum $(5 \mathrm{mM})$ were kept constant while bicarbonate and calcium served as experimental variables with high $(50 \mathrm{mM}$ and $5 \mathrm{mM})$ and low $(3 \mathrm{mM}$ and $0 \mathrm{mM})$ concentrations. Stock pore water solutions were prepared for 
each sample set using combinations of various concentrated salt solutions formulated in a way such that the final concentrations of the primary components of interest would be within the desired range when made up to volume.

Initial sample sets were comprised of four synthetic pore water solutions, prepared identically with the exception of the two variables being evaluated, bicarbonate $\left(\mathrm{HCO}_{3}{ }^{-}\right)$ and calcium $(\mathrm{Ca})$. In order to observe the impact that these variables had on the uranium phase being produced with the application of the remediation method, synthetic pore water sample solutions were formulated to have varying combinations of high and low bicarbonate concentrations, with and without the presence of calcium (Table 1).

Table 1. Stock Solutions and Sample Mixtures

\begin{tabular}{|c|c|c|c|c|c|}
\hline \multirow[b]{2}{*}{ Stock Solution } & \multirow[b]{2}{*}{$\begin{array}{c}\text { Stock Solution } \\
\text { Concentration } \\
\qquad(\mathrm{mM})\end{array}$} & \multicolumn{4}{|c|}{ Synthetic Pore Water Concentrations (mM) } \\
\hline & & $\begin{array}{c}\text { Low } \\
\text { bicarbonate } \\
\text { w/o calcium }\end{array}$ & $\begin{array}{c}\text { Low } \\
\text { bicarbonate } \\
\text { w/ calcium }\end{array}$ & $\begin{array}{c}\text { High } \\
\text { bicarbonate } \\
\text { w/o calcium }\end{array}$ & $\begin{array}{c}\text { High } \\
\text { bicarbonate } \\
\text { w/ calcium }\end{array}$ \\
\hline $\mathrm{CaCl}_{2} \cdot 2 \mathrm{H}_{2} \mathrm{O}$ & 147.01 & 0 & 5 & 0 & 5 \\
\hline $\mathrm{KHCO}_{3}$ & 400.00 & \multicolumn{2}{|c|}{3} & \multicolumn{2}{|c|}{50} \\
\hline $\mathrm{Na}_{2} \mathrm{SiO}_{3} \cdot 9 \mathrm{H}_{2} \mathrm{O}$ & 50 & \multicolumn{4}{|c|}{100} \\
\hline $\mathrm{Al}\left(\mathrm{NO}_{3}\right)_{3}$ & 422.24 & \multicolumn{4}{|c|}{5} \\
\hline $\mathrm{UO}_{2}\left(\mathrm{NO}_{3}\right)_{2} \cdot 6 \mathrm{H}_{2} \mathrm{O}$ & 4.2 & \multicolumn{4}{|c|}{$0.84(\sim 200 \mathrm{ppm})$} \\
\hline
\end{tabular}

The sample preparation process began with the preparation of concentrated salt solutions of $\mathrm{KHCO}_{3}, \mathrm{Na}_{2} \mathrm{SiO}_{3}$, and $\mathrm{Al}\left(\mathrm{NO}_{3}\right)_{3}$, which were then combined in a $50 \mathrm{~mL}$ vial at the ratios required to achieve the desired final concentrations when made up to volume with deionized water. Initially, only two of these mixed solutions were prepared; one for each of the two $\mathrm{HCO}_{3}{ }^{-}$concentrations being evaluated. Later in the process, these solutions would be divided into their smaller, labeled containers before adding their calcium and uranium components in the final step. The late addition of uranium and calcium was intended to avoid possible errant side product formations in the synthetic pore water 
preparation. The solutions, which are relatively alkaline at this point, were adjusted to a $\mathrm{pH}$ of 8 , using concentrated nitric acid, to bring the solution down to a $\mathrm{pH}$ that better represents the natural $\mathrm{pH}$ conditions of the Hanford 200 Area vadose zone (Serne et al., 2008; Szecsody et al., 2012). After the $\mathrm{pH}$ adjustment, prior to the addition of the calcium and uranium components, the remediation method was applied by basifying the solution by sparging ammonia $\left(\mathrm{NH}_{3}\right)$ gas until the $\mathrm{pH}$ range of 11-12 was achieved. From there, 10 $\mathrm{mL}$ aliquots of the $50 \mathrm{~mL}$ stock were dispensed into $15 \mathrm{~mL}$ sample vials, one for each calcium concentration. Solutions were finished by adding concentrated $\mathrm{CaCl}_{2}$ and $\mathrm{UO}_{2}\left(\mathrm{NO}_{3}\right)_{2}$ solutions up to concentration.

With the addition of the final synthetic pore water components, the mixture was allowed a development period to allow for the equilibration and stabilization of the supernatant and precipitate phases. The development period was defined as the time between the addition of the final synthetic pore water component (uranyl nitrate) and the separation of the precipitate and supernatant. A time study was done to investigate how the development period affected the production of the solid uranyl analyte. For this time study, the four basic synthetic pore water solutions, using combinations of high and low calcium and bicarbonate concentrations, described in Table 1, were prepared in quintuplets to allow each four-sample set a development period between 2 days and 3 months (Table 2). 
Table 2. Sample Labels with Development Time

\begin{tabular}{|c|c|c|c|c|c|c|}
\hline \multicolumn{2}{|c|}{$\begin{array}{l}\text { High (+) or Low (-) } \\
\text { Concentration }\end{array}$} & \multicolumn{5}{|c|}{ Equilibration/Development Time } \\
\hline [Bicarbonate] & [Calcium] & 2 Days & 2 Weeks & 1 Month & 2 Months & 3 Months \\
\hline$x^{2}+5$ & $x^{-2}+5$ & $\begin{array}{l}\text { LBi-LCa- } \\
\text { 2D }\end{array}$ & $\begin{array}{l}\text { LBi-LCa- } \\
2 \mathrm{~W}\end{array}$ & $\begin{array}{l}\text { LBi-LCa- } \\
1 \mathrm{M}\end{array}$ & $\begin{array}{l}\text { LBi-LCa- } \\
2 \mathrm{M}\end{array}$ & $\begin{array}{l}\text { LBi-LCa- } \\
3 \mathrm{M}\end{array}$ \\
\hline+ & - & $\begin{array}{l}\mathrm{HBi}-\mathrm{LCa}- \\
\text { 2D }\end{array}$ & $\begin{array}{l}\text { HBi-LCa- } \\
2 \mathrm{~W}\end{array}$ & $\begin{array}{l}\text { HBi-LCa- } \\
\text { 1M }\end{array}$ & $\begin{array}{l}\mathrm{HBi}-\mathrm{LCa}- \\
2 \mathrm{M}\end{array}$ & $\begin{array}{l}\text { HBi-LCa- } \\
\text { 3M }\end{array}$ \\
\hline- & + & $\begin{array}{l}\mathrm{LBi}-\mathrm{HCa}- \\
\text { 2D }\end{array}$ & $\begin{array}{l}\text { LBi-HCa- } \\
2 \mathrm{~W}\end{array}$ & $\begin{array}{l}\text { LBi-HCa- } \\
1 \mathrm{M}\end{array}$ & $\begin{array}{l}\text { LBi-HCa- } \\
2 \mathrm{M}\end{array}$ & $\begin{array}{c}\mathrm{LBi}-\mathrm{HCa}- \\
3 \mathrm{M}\end{array}$ \\
\hline+ & + & $\begin{array}{l}\mathrm{HBi}-\mathrm{HCa}- \\
\text { 2D }\end{array}$ & $\begin{array}{l}\mathrm{HBi}-\mathrm{HCa}- \\
2 \mathrm{~W}\end{array}$ & $\begin{array}{l}\mathrm{HBi}-\mathrm{HCa}- \\
1 \mathrm{M}\end{array}$ & $\begin{array}{l}\mathrm{HBi}-\mathrm{HCa}- \\
2 \mathrm{M}\end{array}$ & $\begin{array}{c}\mathrm{HBi}-\mathrm{HCa}- \\
3 \mathrm{M}\end{array}$ \\
\hline
\end{tabular}

The precipitate was separated by manually decanting the supernatant; working cautiously to avoiding disturbing the settled solids. The isolated supernatant solution was reserved for analysis via KPA while the solid was dried in an oven at $30^{\circ} \mathrm{C}$ for two weeks. A portion of each of the dried samples precipitates would be taken for SEM-EDS and XRD analysis.

My initial investigation included several variations of the aforementioned sample preparation procedure. These exploratory changes were used to further develop the initial sample preparation method prior to getting into the principal experimental study. Chief among these deviations was the elevation of uranium concentration in the synthetic pore water solutions from $200 \mathrm{ppm}(0.84 \mathrm{mM})$ to $500 \mathrm{ppm}(2.1 \mathrm{mM})$, detailed in 4.1 - Synthetic Pore Water System Optimization. Though the variety of changes examined were valuable in these introductory investigations, the extent to which they were analyzed, and thus reported, varied according to their respective pertinence to the primary investigation.

\subsection{Surface Analysis by SEM-EDS}

Scanning electron microscopy with energy dispersive spectroscopy (SEM-EDS) of the dried precipitate sample surface was used as the focal point of the early analysis of the 
experimental methodology. Through its electron-sample interactions, the technique provided valuable information on the surface morphology and chemical composition of samples. Of particular use was the ability to use backscatter electron detection to quickly, visually differentiate areas of higher average atomic number which, in these samples, would likely be indicative of an area rich in uranium, the analyte of interest.

\subsubsection{Methods}

Prior to analysis, small specimens were taken from each of the solid precipitate samples and mounted to individual aluminum studs using a conductive double-sided carbon tape (Figure 1). In order to ensure a conductive sample surface, all samples were coated using an SPI-Module Control and Sputter unit for 1-2 minutes to produce a conductive gold layer. The gold coating helps to minimize sample charging, which occurs when electrons collect and build up a charge on the sample surface, causing unexpected effects and visual artifacts.

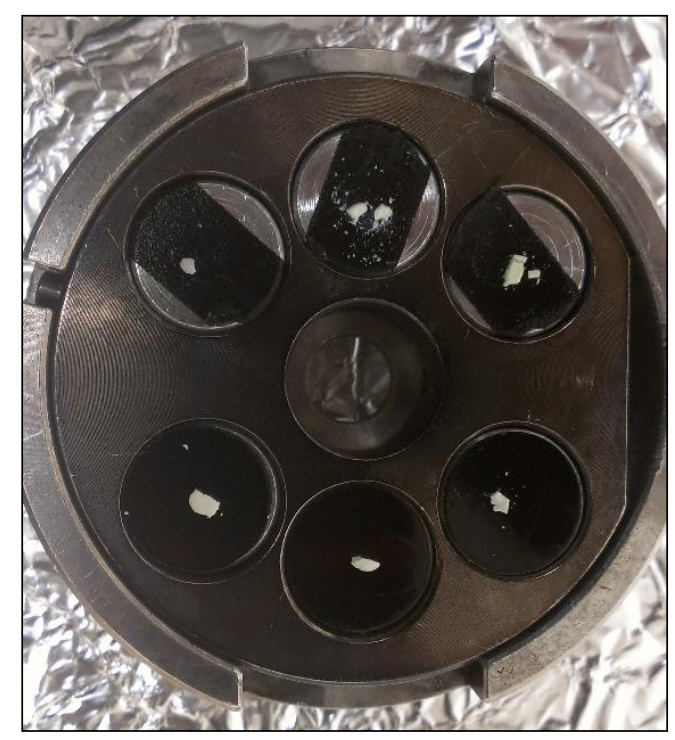

Figure 1. Precipitates loaded on studs using carbon tape and mounted for SEM 
The system used for this analysis, provided by the Florida Center for Analytical Electron Microscopy (FCAEM) on the Florida International University Modesto A. Maidique Campus, was a JOEL-5910-LV scanning electron microscope equipped with an EDAX Sapphire detector with UTW Window for EDS analysis. Throughout the analysis, the instrument was set up to use acceleration potentials ranging from 10 to $20 \mathrm{kV}$ and spot sizes ranging from 30 to $45 \mu \mathrm{m}$. Though some imaging was done in secondary electron mode, the majority of micrographs were collected using backscatter electron capture mode in order to better target the areas of higher average atomic mass. These regions, indicated by the lighter shading, were targeted compositional analysis by EDS in order to confirm the presence of uranium, the analyte of particular interest in the present study.

\subsubsection{Results and Discussion}

For the preliminary look at sample precipitates prepared using the experimental remediation method, the focal point of the investigation was the qualitative morphological analysis and the semi-quantitative elemental analysis provided by scanning electron microscopy and energy dispersive spectroscopy, respectively. The results would be used to make adjustments to the sample preparation methods while contributing evidence towards the overall sample characterization effort. On the basis of previous reports (Lagos et al., 2012), it was anticipated that sample precipitates would have crystalline structures which EDS would confirm to be relatively rich in uranium. For EDS analysis, a uranium atomic percentage of $1 \%$ was arbitrarily assigned as the threshold for what was considered significant or uranium-rich. As previously explained, these structures should be distinguishable by their brightness in the SEM's backscatter electron capture mode, 
because of the increased elastic collisions attributed to uranium's significantly larger atomic number and size.

It was hypothesized that there would be an inverse relationship between the bicarbonate concentration in the synthetic pore water solution and the uranium that precipitated. With decreasing bicarbonate in solution, it was anticipated that the uranium in precipitate would increase as a result of a decrease in the formation of soluble uranyl carbonate species. Following this prediction, it was expected that the low bicarbonate precipitate samples would bear the most uranium solids for detection and analysis. Despite that expectation, the SEM-EDS analysis of low the carbonate samples, both with and without calcium, revealed virtually no uranium-rich regions.

Analysis of these low bicarbonate samples include SEM images with accompanying EDS data corresponding to points of analysis targeting areas of particularly intriguing formations (Figure $2 \&$ Figure 3). Generally, the bulk of these samples would be made up of either the rough, brittle-seeming surfaces observed in the low (zero) calcium sample displayed in Figure 2, the smooth, curved surfaces observed in the high $(5 \mathrm{mM})$ calcium sample presented in Figure 3, or both. Comparison to other samples shows that this quality has no apparent connection to the calcium content. 


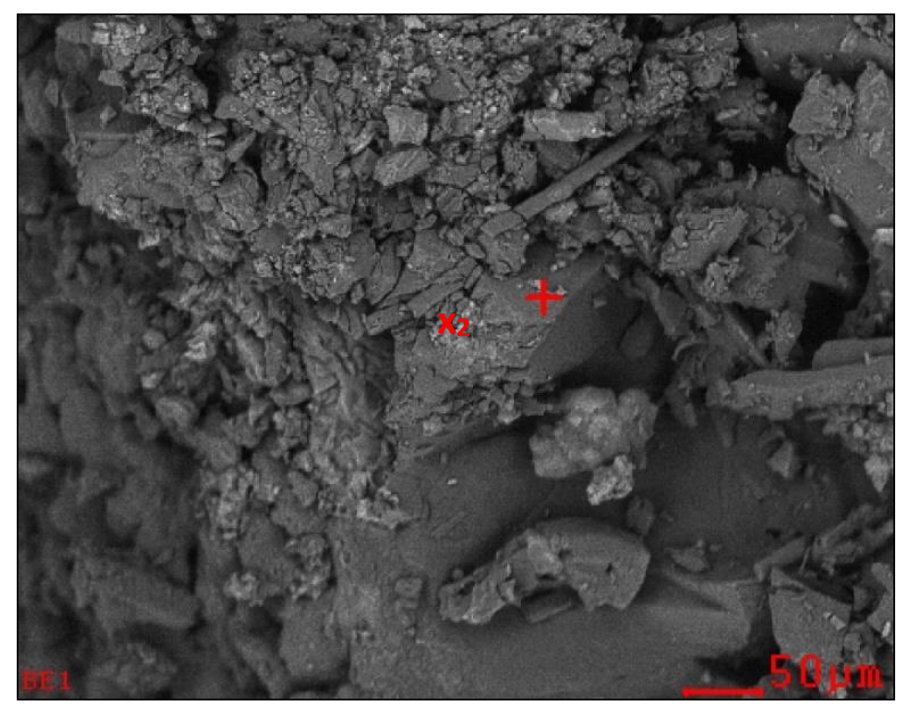

\begin{tabular}{|c|c|c|c|}
\hline \multicolumn{2}{|c|}{$\mathrm{X}_{1}$} & \multicolumn{2}{|l|}{ X } \\
\hline Element & At\% & Element & At\% \\
\hline $\mathbf{C}-\mathbf{K}_{\boldsymbol{a}}$ & 10.07 & $\mathbf{C}-\mathbf{K}_{\boldsymbol{a}}$ & 15.86 \\
\hline $\mathbf{N}-\mathbf{K}_{\boldsymbol{\alpha}}$ & 18.79 & $-\mathbf{K}_{\boldsymbol{\alpha}}$ & 11.27 \\
\hline $\mathbf{O}-\mathbf{K}_{\boldsymbol{a}}$ & 44.70 & $-\mathbf{K}_{\boldsymbol{\alpha}}$ & 47.64 \\
\hline $\mathbf{N a}-\mathbf{K}_{\boldsymbol{\alpha}}$ & 24.9 & $-\mathbf{K}_{\boldsymbol{\alpha}}$ & 07.16 \\
\hline Al & 00 & $\mathbf{K}_{\boldsymbol{a}}$ & 00. \\
\hline $\mathbf{S i}$ & 01 . & $\mathbf{K}_{\boldsymbol{\alpha}}$ & 16.84 \\
\hline $\mathbf{K}_{\boldsymbol{a}}$ & 00 . & $\mathbf{C l}-\mathbf{K}_{\boldsymbol{\alpha}}$ & 00.11 \\
\hline $\mathbf{U}-\mathbf{M}_{\boldsymbol{\alpha}}$ & 00.1 & $\mathbf{U}-\mathbf{M}_{\boldsymbol{\alpha}}$ & 00.20 \\
\hline $\mathbf{K}-\mathbf{K}_{\boldsymbol{\alpha}}$ & 00.00 & $\mathbf{K}-\mathbf{K}_{\boldsymbol{a}}$ & 00.23 \\
\hline $\mathbf{C a}-\mathbf{K}_{\boldsymbol{\alpha}}$ & 00.07 & $\mathbf{C a}-\mathrm{K}_{\alpha}$ & 00.06 \\
\hline
\end{tabular}

Figure 2. SEM image and EDS data for a $3 \mathrm{mM}$ bicarbonate, $0 \mathrm{mM}$ calcium sample

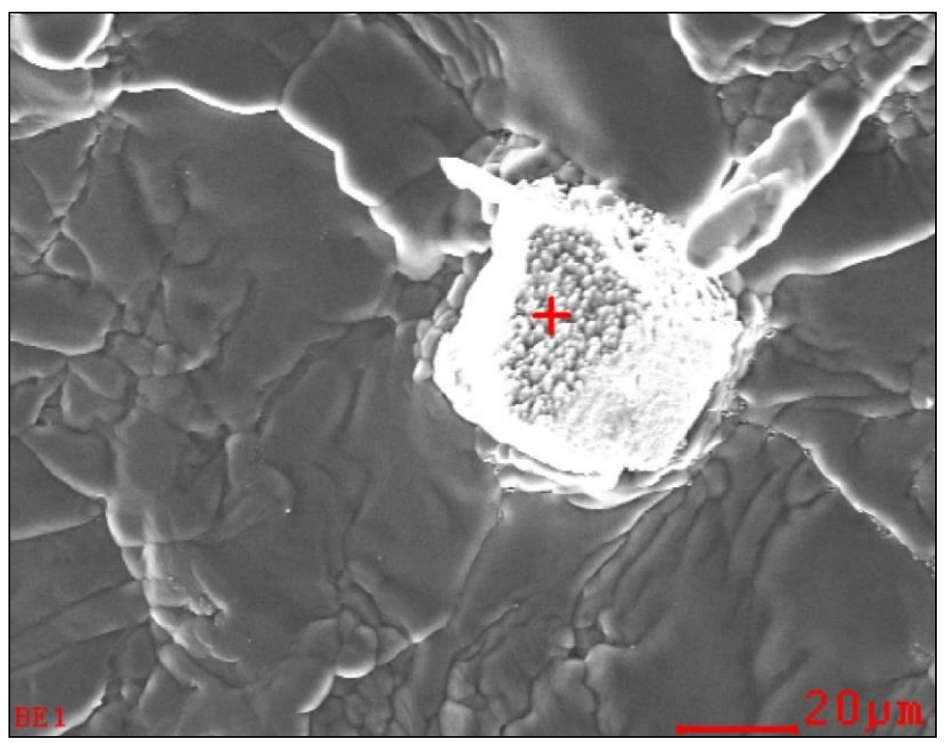

\begin{tabular}{|c|c|}
\multicolumn{1}{c}{$\mathrm{X}_{1}$} \\
\hline Element & $\mathbf{A t} \%$ \\
\hline $\mathbf{C}-\mathbf{K}_{\boldsymbol{\alpha}}$ & 21.90 \\
$\mathbf{N}-\mathbf{K}_{\boldsymbol{\alpha}}$ & 06.40 \\
$\mathbf{O}-\mathbf{K}_{\boldsymbol{\alpha}}$ & 53.20 \\
$\mathbf{N a}-\mathbf{K}_{\boldsymbol{\alpha}}$ & 00.29 \\
$\mathbf{A l}-\mathbf{K}_{\boldsymbol{\alpha}}$ & 00.00 \\
$\mathbf{S i}-\mathbf{K}_{\boldsymbol{\alpha}}$ & 00.32 \\
$\mathbf{C l}-\mathbf{K}_{\boldsymbol{\alpha}}$ & 00.03 \\
$\mathbf{U}-\mathbf{M}_{\boldsymbol{\alpha}}$ & 00.04 \\
$\mathbf{K}-\mathbf{K}_{\boldsymbol{\alpha}}$ & 00.02 \\
$\mathbf{C a}-\mathbf{K}_{\boldsymbol{u}}$ & 17.77 \\
\hline
\end{tabular}

Figure 3. SEM image and EDS data for a $3 \mathrm{mM}$ bicarbonate, $5 \mathrm{mM}$ calcium sample

The brighter than average areas were considered most likely to be rich in uranium and were thus the focus of the surface examination. Despite that expectation, the majority of conspicuous regions were analyzed by EDS without regard for their hue. Regions displayed in the aforementioned figures were some of the most intriguing observations in the low bicarbonate samples, and yet, contained minimal amounts of the uranium analyte of interest. Rather, the majority of interesting sites on the low bicarbonate - low calcium 
samples were predominantly made up of carbon, oxygen, nitrogen, sodium, and silicon. Within the low bicarbonate - high calcium samples, highlighted, cubical structures predominantly made up of carbon, nitrogen, oxygen, and calcium, stood apart from the rest of the otherwise dull morphology. Most likely, the latter of those two figures shows a calcium carbonate solid form that precipitated in the sample preparation process.

Unlike their low bicarbonate counterparts, all of the precipitates produced from high bicarbonate synthetic pore water solutions showed areas with relatively high concentrations of uranium. The EDS analysis of the areas that visually stood apart, either because of distinctive formation or brightness, from the bulk of the sample consistently revealed uranium atomic percentages that exceeded 1\% (Figure $4 \&$ Figure 5). Though the morphologies of these uranium-rich areas varied from sample to sample, the most prominent form was the needle-like solids displayed in Figure 5. The EDS analysis for that structure, labeled $\mathrm{X}_{1}$, showed that the major contributing elements in the structure to be oxygen $(49 \%)$, carbon $(\sim 20 \%)$, sodium $(\sim 16 \%)$, nitrogen $(\sim 12 \%)$, and uranium $(\sim 2 \%)$. Conversely, the uranium-rich phase observed in Figure 4 does not show such a clear structure, despite being richer than average in uranium $(\sim 5 \%)$. The distinction between these samples could be related to the difference in calcium in their synthetic pore water base solutions or a physical change related to the influence of the silica rich solid (11-18\%) in which the uranium-bearing solid is embedded. 


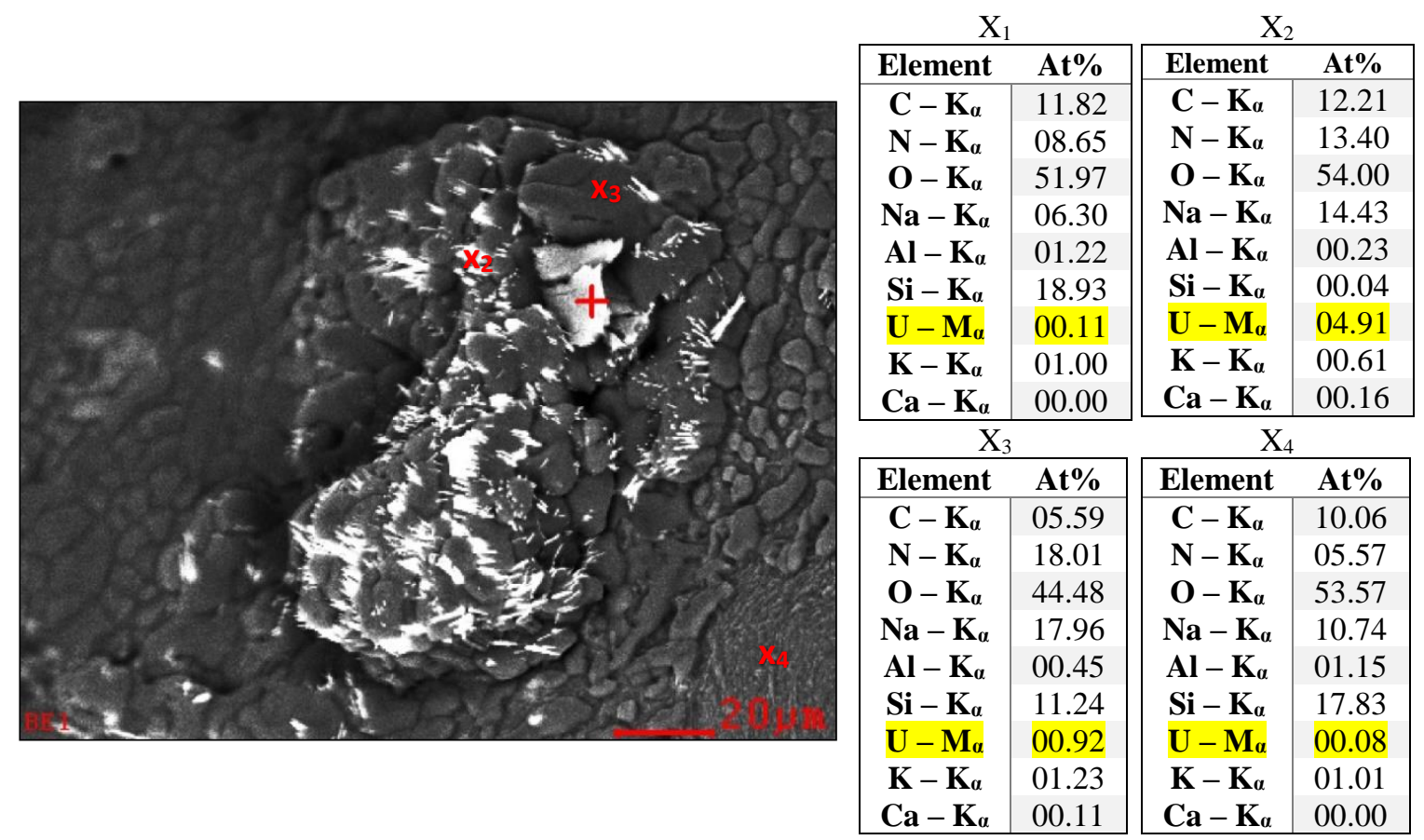

Figure 4. SEM image and EDS data for a $50 \mathrm{mM}$ bicarbonate, $0 \mathrm{mM}$ calcium sample

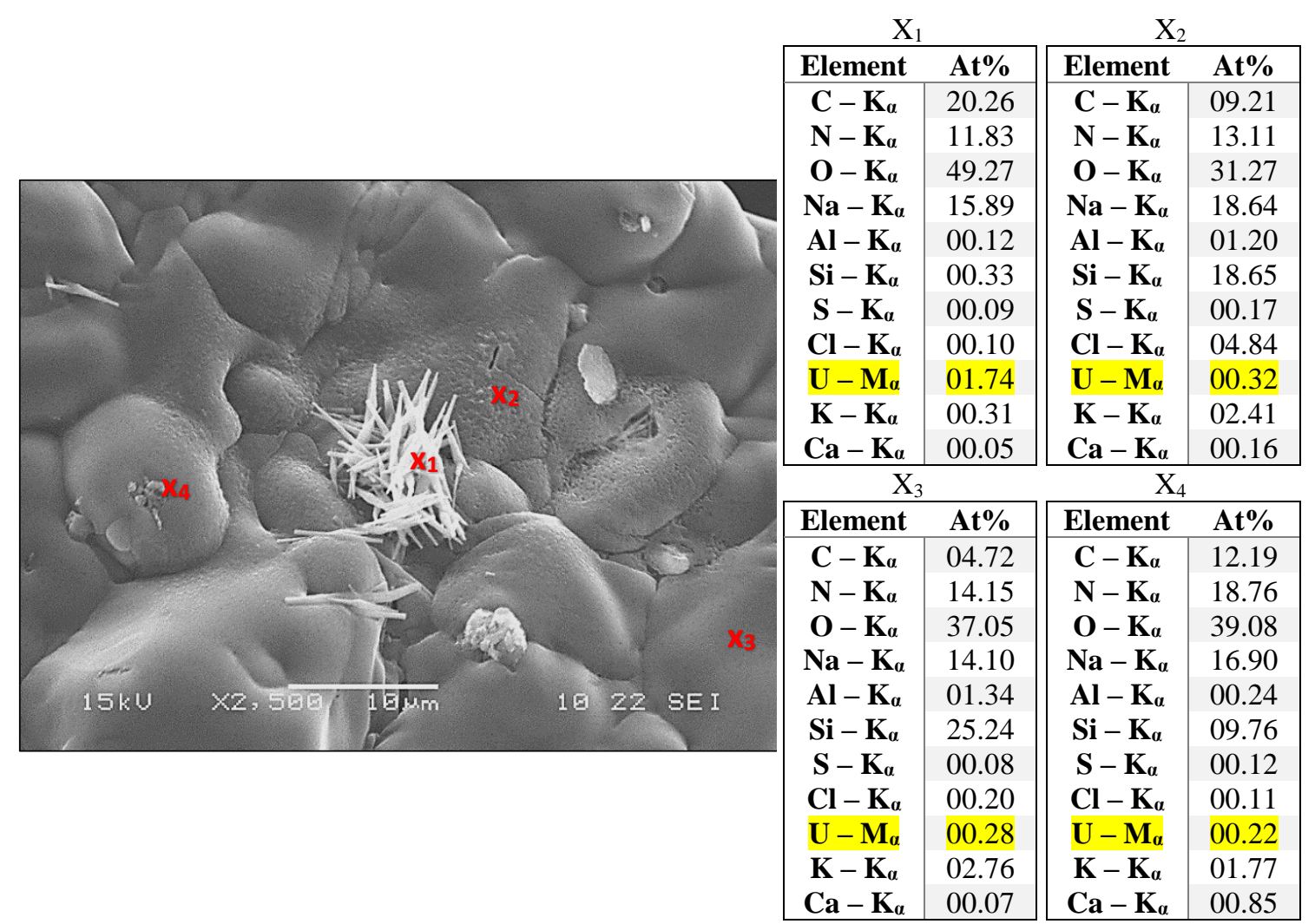

Figure 5. SEM image and EDS data for a $50 \mathrm{mM}$ bicarbonate, $5 \mathrm{mM}$ calcium sample 
Like the results of low bicarbonate sample analysis, these findings disagree with the prediction that increasing the bicarbonate concentration in synthetic pore water solutions would result in an increase in soluble uranium-bearing species. To the contrary, nearly all high bicarbonate samples showed regions which EDS analysis would determine to be uranium-rich. The lack of uranium-rich regions in the low bicarbonate samples, along with the limited access to instrumentation and facilities suitable or amenable to working with samples which contained a radionuclide like uranium, led to the decision to limit further analysis to the high bicarbonate samples which had already been proven to bear uranium precipitates.

The time study, used to probe the relationship between the development time in solution and the precipitation of the targeted uranyl-rich analyte, relied on the qualitative and semiquantitative results of SEM and EDS across the array of samples. Considering the dearth of uranium products observed in the early investigation of the low bicarbonate samples and the consistency with which such forms were observed on the high bicarbonate variety, it was determined that resources would be best used by modifying the time study to limit it to the high bicarbonate samples (Table 3). The prudent decision allowed for a more thorough investigation and comparison of the most relevant samples while further helping to scrutinize and advance the development of the sample preparation methods leading into the principal study. 
Table 3. Condensed Series of Samples with Development Time

\begin{tabular}{|c|c|c|c|c|c|c|}
\hline \multicolumn{2}{|c|}{$\begin{array}{c}\text { High (+) or Low (-) } \\
\text { Concentration }\end{array}$} & \multicolumn{5}{c|}{ Equilibration/Development Time } \\
\hline [Bicarbonate] & [Calcium] & $\mathbf{2}$ Days & $\mathbf{2}$ Weeks & $\mathbf{1}$ Month & $\mathbf{2}$ Months & $\mathbf{3}$ Months \\
\hline $\boldsymbol{t}$ & - & $\mathrm{HBi}-\mathrm{LCa}-$ & $\mathrm{HBi}-\mathrm{LCa}-$ & $\mathrm{HBi}-\mathrm{LCa}-$ & $\mathrm{HBi}-\mathrm{LCa}-$ & $\mathrm{HBi}-\mathrm{LCa}-$ \\
& & $2 \mathrm{D}$ & $2 \mathrm{~W}$ & $1 \mathrm{M}$ & $2 \mathrm{M}$ & $3 \mathrm{M}$ \\
\hline+ & + & $\mathrm{HBi}-\mathrm{HCa}-$ & $\mathrm{HBi}-\mathrm{HCa}-$ & $\mathrm{HBi}-\mathrm{HCa}-$ & $\mathrm{HBi}-\mathrm{HCa}-$ & $\mathrm{HBi}-\mathrm{HCa}-$ \\
& & $2 \mathrm{D}$ & $2 \mathrm{~W}$ & $1 \mathrm{M}$ & $2 \mathrm{M}$ & $3 \mathrm{M}$ \\
\hline
\end{tabular}

Throughout the time study, all high bicarbonate samples were revealed to have regions fitting the uranium-rich description with atomic percentages exceeding 1\% (Figure $6 \&$ Figure 7). For the majority of samples, the uranium was concentrated in the needle-like structures which were highlighted in backscatter electron capture mode. In terms of attributes such as thickness and cluster density, these forms were relatively consistent throughout each individual sample. The presence of this consistent form supported a working assumption that these structures were in fact repeating crystalline patterns. Though these needle-like crystals were fairly homogenous within each sample, there was significant variation in the precipitates across the timeline.

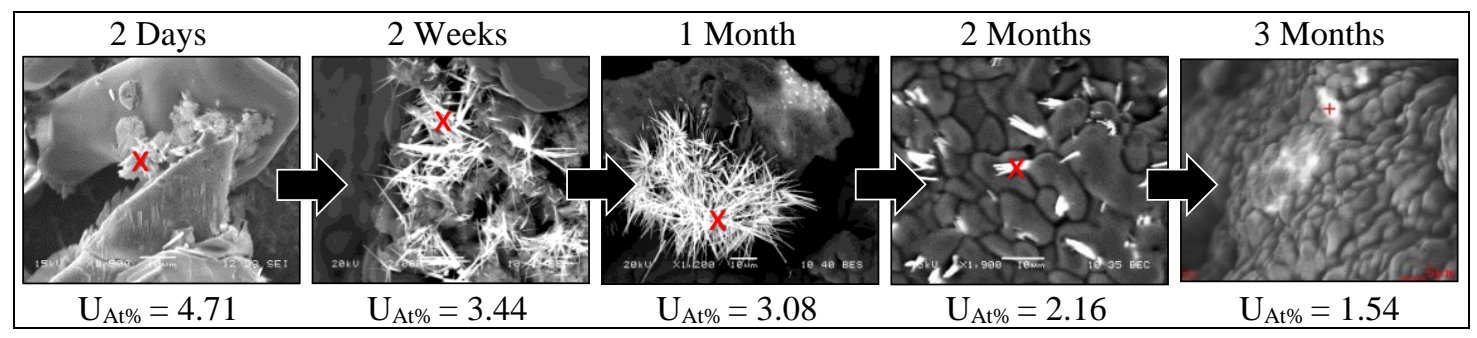

Figure 6. Timeline of uranium-rich region change with increasing development time in $\mathrm{HBi}-\mathrm{LCa}$ samples

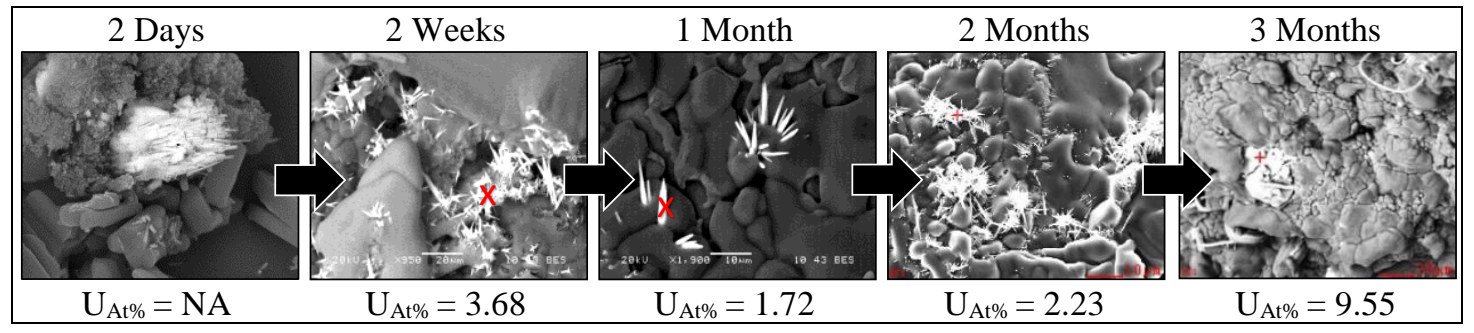

Figure 7. Timeline of uranium-rich region change with increasing development time in $\mathrm{HBi}-\mathrm{HCa}$ samples 
Of the multitude of points tested, a comparison of some of the highest uranium atomic percentages $\left(\mathrm{U}_{\mathrm{At}} \%\right.$ ) on each sample found what appeared to be a decreasing trend with time in the HBi-LCa series (Figure 6). This decreasing trend, where less uranium is found in the precipitate phase with increasing time in solution, could be related to a change in the type of uranium solids observed in the micrographs for the shorter developing samples like HBiLCa-2D. The idea is supported by the diminishing of the of the needle-like clusters, from their prominence in the 1-month precipitate to the complete lack of any discernably crystalline forms in the 3-month.

Unlike the HBi-LCa series, there was no evident correlation between uranium content in the precipitates and the time in solution. Though the $\mathrm{U}_{\mathrm{At}} \%$ was omitted for the $\mathrm{HBi}-\mathrm{HCa}-$ 2D precipitate sample because of an error in EDS analysis drastically skewing the value, it was apparent that the abundance trended down from that 2-day sample to the 1-month sample and back up from the 1-month to the 3-month sample. Like the uranium abundance, there was no clear progression in the morphological characteristics of the uranium-rich crystalline regions across the range of the time study. The array of SEM images reveal significant sample-to-sample diversity in the shape and cluster size of these points of interest with no evident trend.

Interestingly, in both series, the samples prepared using the 3-month development period were the only ones not to show the needle-shaped uranium-rich precipitates. Rather, the uranium analyte was found collected in less definitive formations; physically appearing to be highly incorporated into the bulk of the solid as opposed to standing out like the easily distinguishable needle-like clusters. The lack of definition compared to that observed in 
samples like $\mathrm{HBi}-\mathrm{HCa}-2 \mathrm{~W}$ (Figure 7), suggests that these solid uranium-rich species are likely significantly different, and possibly amorphous. Though not morphologically identical, this seemingly amorphous type of uranium-bearing solid was also observed in the HBi-HCa-1M sample (Figure 8). What is unique about this 1-month sample, compared to the 3-month samples, is the apparent formation of the same needle-like crystals characteristic of the low development time samples which formed alongside the amorphous region. The fact that these types of phases were most prominently observed on the 3-month samples could be indication of some type of change associated with the development time. This could suggest that the intermediate $\mathrm{HBi}-\mathrm{HCa}-1 \mathrm{M}$ sample could represent a transition period between the two types of phases wherein both are present. The overall meager abundance of uranium-rich areas in these samples is likely also associated with the extended development period.

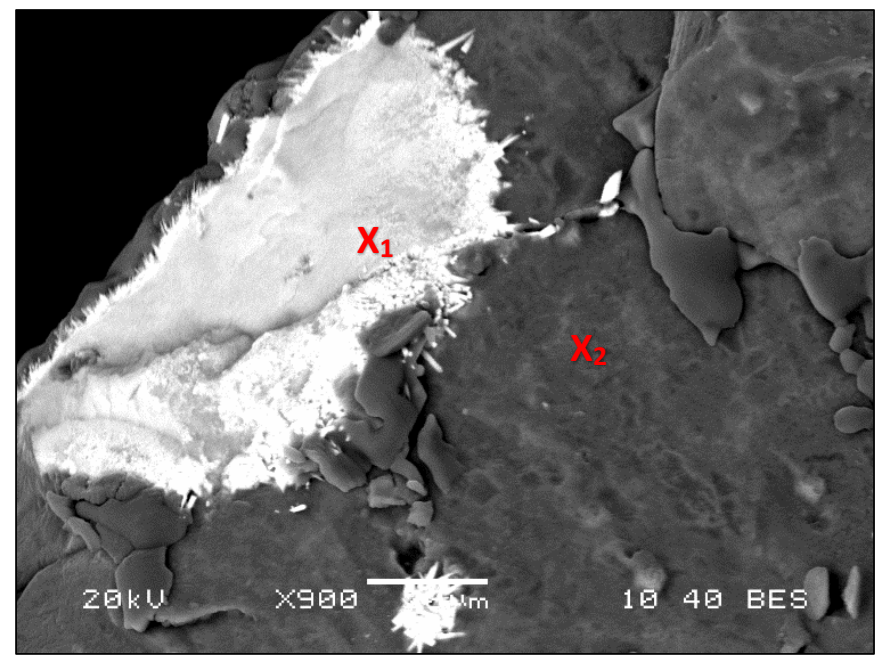

\begin{tabular}{|c|c|c|c|}
\hline \multicolumn{2}{|c|}{$X_{1}$} & \multicolumn{2}{|c|}{$\mathrm{X}_{2}$} \\
\hline Element & At $\%$ & Element & At $\%$ \\
\hline $\mathbf{C}-\mathbf{K}_{\boldsymbol{\alpha}}$ & 11.34 & $\mathbf{C}-\mathbf{K}_{\boldsymbol{a}}$ & 05.61 \\
\hline $\mathbf{N}-\mathbf{K}_{\boldsymbol{\alpha}}$ & 07.27 & $\mathbf{N}-\mathbf{K}_{\boldsymbol{\alpha}}$ & 10.97 \\
\hline $\mathbf{O}-\mathbf{K}_{\boldsymbol{a}}$ & 55.30 & $\mathbf{O}-\mathbf{K}_{\boldsymbol{\alpha}}$ & 50.25 \\
\hline $\mathbf{N a}-\mathbf{K}_{\boldsymbol{\alpha}}$ & 12.60 & $\mathbf{N a}-\mathbf{K}_{\boldsymbol{\alpha}}$ & 10.14 \\
\hline $\mathbf{A l}-\mathbf{K}_{\boldsymbol{\alpha}}$ & 00.63 & $\mathbf{A l}-\mathbf{K}_{\boldsymbol{\alpha}}$ & 01.09 \\
\hline $\mathbf{S i}-\mathbf{K}_{a}$ & 08.72 & $\mathbf{S i}-\mathbf{K}_{\boldsymbol{a}}$ & 15.04 \\
\hline $\mathbf{C l}-\mathbf{K}_{\alpha}$ & 00.44 & $\mathbf{C l}-\mathbf{K}_{\boldsymbol{a}}$ & 00.35 \\
\hline $\mathbf{U}-\mathbf{M}_{\boldsymbol{\alpha}}$ & 01.97 & $\mathbf{U}-\mathbf{M}_{\boldsymbol{\alpha}}$ & 00.13 \\
\hline $\mathbf{K}-\mathbf{K}_{\boldsymbol{a}}$ & 01.69 & $\mathbf{K}-\mathbf{K}_{\boldsymbol{\alpha}}$ & 06.41 \\
\hline $\mathbf{C a}-\mathbf{K}_{\boldsymbol{\alpha}}$ & 00.03 & $\mathbf{C a}-\mathbf{K}_{\alpha}$ & 00.00 \\
\hline
\end{tabular}

Figure 8. SEM image and EDS data for the $50 \mathrm{mM}$ bicarbonate, $5 \mathrm{mM}$ calcium sample with a 1-month development time

Though curious, the relatively low abundance of uranium-rich regions of interest in samples prepared with the longer development times is detrimental to the proposed 
subsequent structural analysis. Samples earlier in the time study were therefore preferred for continued analysis. In addition to the abundance, the physical structure of the areas of interest in the short-developing samples suggested that they were more likely to bear a discernable uranium crystal structure. In particular, the 2-week samples (Figure 6 \& Figure 7), the investigation of which revealed a wealth of uranium-rich needle-like structures, were isolated for structural analysis using powder x-ray diffraction.

\subsection{XRD Structural Analysis}

Powder x-ray diffraction makes use of the diffraction and constructive interference of monochromatic x-rays directed to a crystalline sample. The method would allow the confirmation of a crystalline phase and, if present, the determination of a diffraction pattern which could matched to known reference patterns. Limited access to the $\mathrm{x}$-ray diffractometer required selective choice of samples for continued analysis. Samples selected to progress from initial surface morphology analysis to structural analysis were chosen in order to take advantage of the significant presence of the uranium-rich structures of interest. In order to focus on those structures, analysis was limited to the two-week samples (HBi-LCa-2W \& HBi-HCa-2W).

\subsubsection{Methods}

Sample preparation began with taking a representative specimen from the two-week samples and carefully pulverizing them using a pestle and mortar to the consistency of a fine powder. In order to compensate for the miniscule amount of precipitate sample produced with this method, samples were loaded into a custom sample holder designed with a shallow reservoir for sparse samples. Analyses were performed using a Bruker 
5000D XRD equipped with a copper $(\mathrm{Cu} \mathrm{K} \alpha)$ radiation source $(\lambda=0.154056 \mathrm{~nm})$ with tungsten filter at $35 \mathrm{kV}$ and $40 \mathrm{~mA}$. For all samples, the sequence was programmed to run over a 2-theta (29) range from $10^{\circ}$ to $75^{\circ}$ with a $0.02^{\circ}$ step size and 2 second counting per step. The resulting diffraction patterns were compared to a library of diffraction patterns using Match! software as well as against the reference patterns for potential mineral forms unavailable in the software library.

\subsubsection{XRD Data \& Analysis}

The x-ray diffraction analysis of select samples served to expand on the SEM-EDS findings by characterizing the likely crystalline structures observed on the sample surface. The resulting data included diffraction patterns with well-defined peaks for all samples evaluated, confirming the presence of crystalline material. Several of the major peaks, include the most prominent at $2 \Theta \approx 29$, were present in all samples regardless of calcium, bicarbonate, or uranium content. The similarities in these patterns suggest the presence of a crystalline solid phase that is consistently present, despite the major differing variables.

In comparing the diffraction patterns of all analyzed samples, nitratine $\left(\mathrm{NaNO}_{3}\right)$ was recognized as the most likely major constituent in all precipitates. Direct comparisons of the sample diffraction patterns with the major peaks for nitratine show that the majority of peaks align well with the reference diffraction pattern for nitratine (Figure 9). The overall intensity of the peaks for the calcium-free and calcium-containing samples is drastically different, with the most prominent one $(2 \Theta \approx 29)$ of the former showing up at three times the intensity of the latter. While the calcium-free sample appears to be a near perfect match, with most significant peaks aligning with those of nitratine, the sample shows peaks at $2 \Theta$ 
$\approx 26.5 \& 47$ not present in nitratine. The calcium-containing sample however show several peaks without corresponding peaks in the nitratine pattern (i.e., $2 \Theta \approx 23.5,32.5,34, \& 41$ ). Assuming that the nitratine identification is accurate, these unmatched peaks could signify the presence of a second crystalline phase. More specifically, they may represent the uranium-containing phases observed in prior SEM/EDS evaluation.
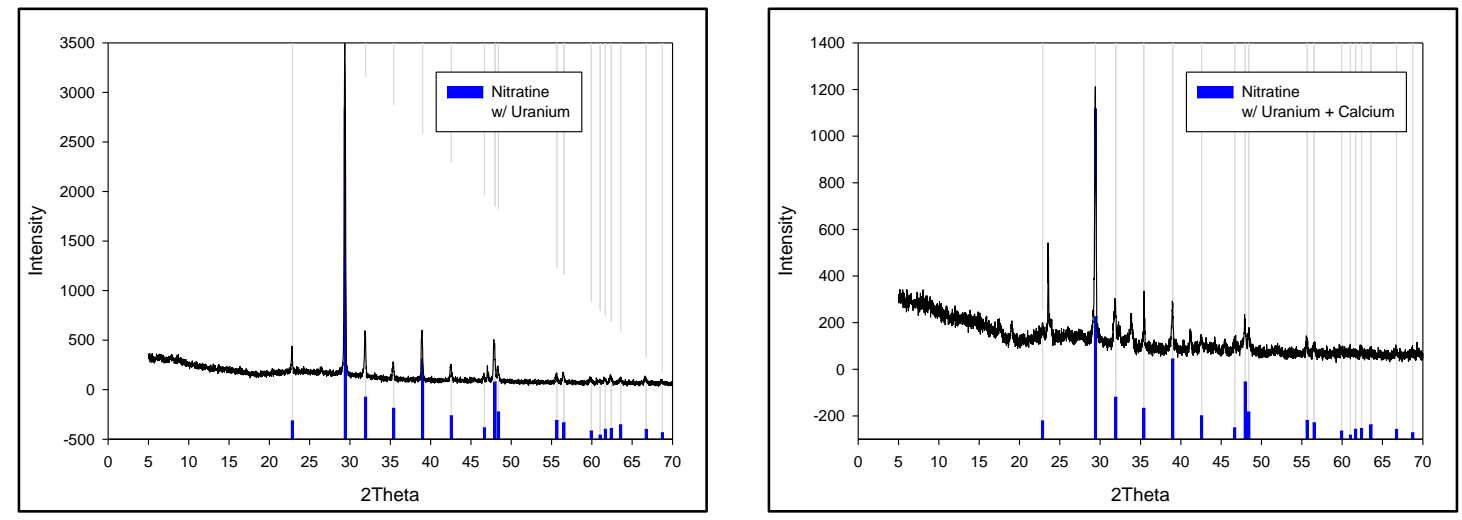

Figure 9. Comparison of the diffraction patterns for the calcium-free (left) and $5 \mathrm{mM}$ calcium-containing (right) precipitate samples with the pattern for nitratine $\left(\mathrm{NaNO}_{3}\right)$

The comparison of the sample diffraction patterns to the anticipated uranium-bearing species yielded no apparent matches. Alternatively, cejkaite $\left(\mathrm{Na}_{4}\left(\mathrm{UO}_{2}\right)\left(\mathrm{CO}_{3}\right)_{3}\right)$ has emerged as a potential match for the uranium-rich phases observed in the SEM/EDS analysis. The comparison of the diffraction patterns revealed that two of the three most prominent peaks $(2 \Theta \approx 17.5 \& 19)$ appear to have a corresponding match in the experimental diffraction patterns for the calcium-containing sample (Figure 10). A match for the third largest cejkaite peak $(2 \Theta \approx 11)$ could not be matched to the diffraction pattern though it is believed to have been concealed by the noisy background. The potential match is complicated by the fact that cejkaite is a highly soluble uranium species that should not precipitate under normal conditions. It is possible that the difficulty in identification comes from the low yield of the uranium phase relative to the bulk of the sample which was noted 
in SEM/EDS analysis. The comparison of the calcium-free sample to the cejkaite XRD pattern showed no apparent peaks at the reference cejkaite $2 \Theta$ values. The failure to match peaks is unsurprising considering that there were few, if any, unmatched peaks of any significant intensity in the comparison with nitratine.

The two potential matches notwithstanding, there are remaining unpaired peaks of significant intensity in the calcium-containing sample (e.g., $2 \Theta \approx 23.5$ ) which had no substantial alignment with the reference patterns for potential species. Assuming that cejkaite and nitratine were indeed present in this sample, these unmatched peaks suggest the presence of additional crystalline phases in the heterogenous sample mix. Despite the change in diffraction patterns associated with the addition of calcium, no calciumcontaining reference patterns showed significant correlation to the peaks of the sample diffraction patterns.
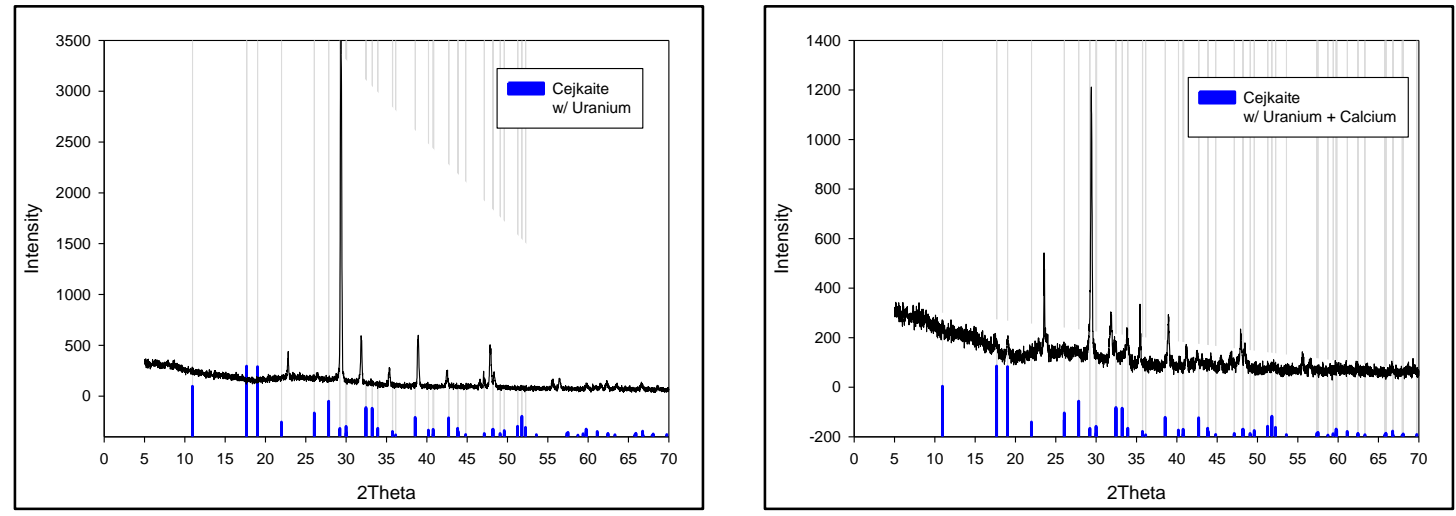

Figure 10. Comparison of the diffraction patterns of calcium-free (left) and $5 \mathrm{mM}$ calcium-containing (right) precipitate samples with the pattern for Cejkaite $\left(\mathrm{Na}_{4}\left(\mathrm{UO}_{2}\right)\left(\mathrm{CO}_{3}\right)_{3}\right)$

The inconclusive results XRD analysis, particularly in the calcium-free sample, prompted a post-analysis re-evaluation of the pulverized sample specimens by scanning electron microscopy with energy dispersive spectroscopy in order to reestablish the presence and physical condition of the uranium-rich regions previously observed. Despite the similar 
findings in the pre-pulverized two-week samples (Figure $6 \&$ Figure 7), the SEM-EDS results of the pulverized sample were starkly different in that no region in the calcium-free sample showed areas of significant uranium accumulation (Figure 11) while the calciumcontaining sample showed several (Figure $12 \&$ Figure 13). These uranium regions included some that distinctly appeared to be fragments of the prominent needle-like structures noted before the sample was ground to powder for analysis (Figure 13). The finding is consistent with the results of the sample diffraction analysis, though the lack of the target analyte in the calcium-free samples disagrees with the initial observations that lead to selecting the sample for continued analysis.

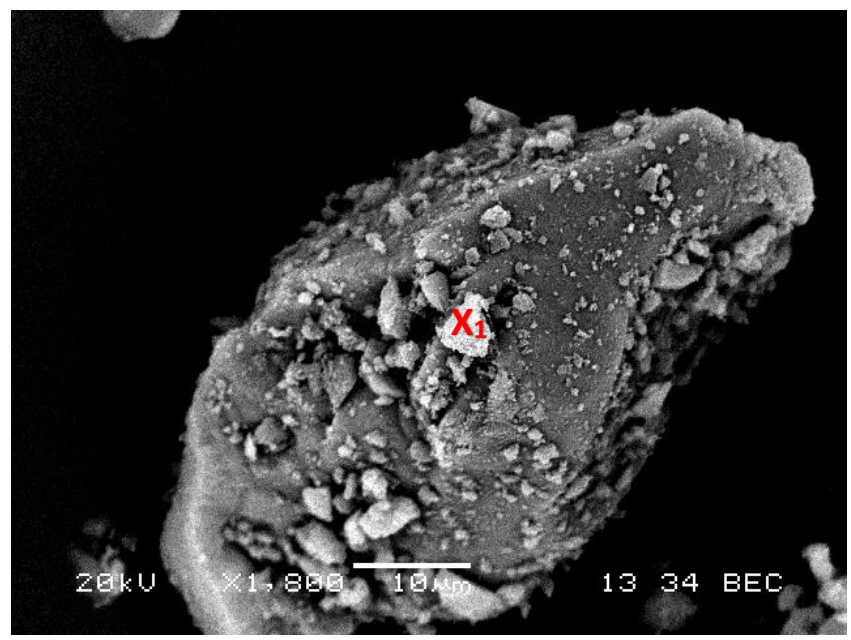

\begin{tabular}{|c|c|}
\multicolumn{1}{c}{$\mathbf{X}_{1}$} \\
\hline Element & $\mathbf{A t} \%$ \\
\hline $\mathbf{C}-\mathbf{K}_{\boldsymbol{\alpha}}$ & 23.20 \\
$\mathbf{N}-\mathbf{K}_{\boldsymbol{\alpha}}$ & 10.90 \\
$\mathbf{O}-\mathbf{K}_{\boldsymbol{\alpha}}$ & 42.80 \\
$\mathbf{N a}-\mathbf{K}_{\boldsymbol{\alpha}}$ & 09.68 \\
$\mathbf{A l}-\mathbf{K}_{\boldsymbol{\alpha}}$ & 00.43 \\
$\mathbf{S i}-\mathbf{K}_{\boldsymbol{\alpha}}$ & 11.84 \\
$\mathbf{C l}-\mathbf{K}_{\boldsymbol{\alpha}}$ & 00.76 \\
$\mathbf{U}-\mathbf{M}_{\boldsymbol{\alpha}}$ & 00.00 \\
$\mathbf{C a}-\mathbf{K}_{\boldsymbol{\alpha}}$ & 00.38 \\
\hline
\end{tabular}

Figure 11. SEM image and EDS data for the ground HBi-LCa-2W sample 


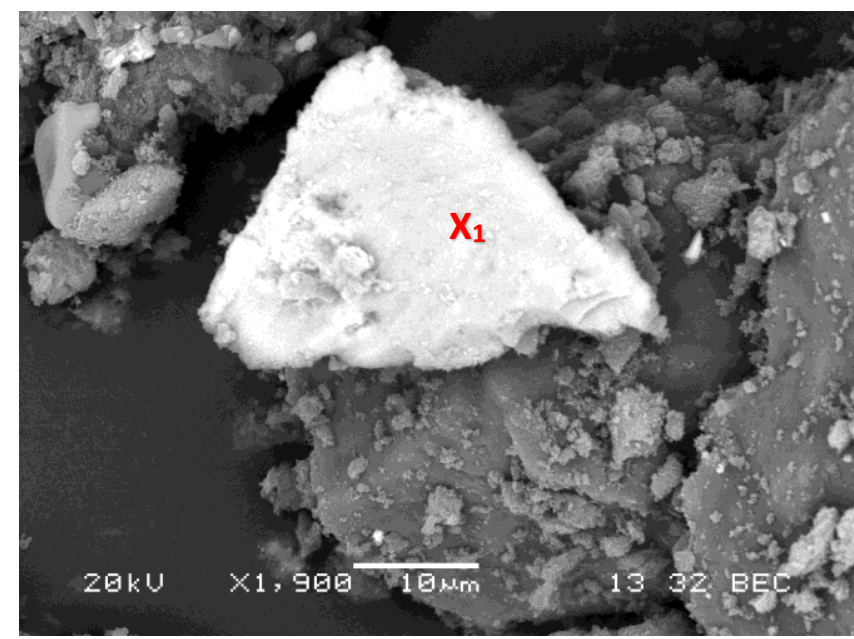

\begin{tabular}{|c|c|}
\multicolumn{2}{c}{$\mathrm{X}_{1}$} \\
\hline Element & $\mathbf{A t} \%$ \\
\hline $\mathbf{C}-\mathbf{K}_{\boldsymbol{\alpha}}$ & 12.01 \\
$\mathbf{O}-\mathbf{K}_{\boldsymbol{\alpha}}$ & 51.80 \\
$\mathbf{N a}-\mathbf{K}_{\boldsymbol{\alpha}}$ & 15.30 \\
$\mathbf{A l}-\mathbf{K}_{\boldsymbol{\alpha}}$ & 00.93 \\
$\mathbf{S i}-\mathbf{K}_{\boldsymbol{\alpha}}$ & 14.68 \\
$\mathbf{U}-\mathbf{M}_{\boldsymbol{\alpha}}$ & 03.34 \\
$\mathbf{K}-\mathbf{K}_{\boldsymbol{\alpha}}$ & 01.94 \\
\hline
\end{tabular}

Figure 12. SEM image and EDS data showing a uranium rich region in the ground $\mathrm{HBi}-\mathrm{HCa}-2 \mathrm{~W}$ sample

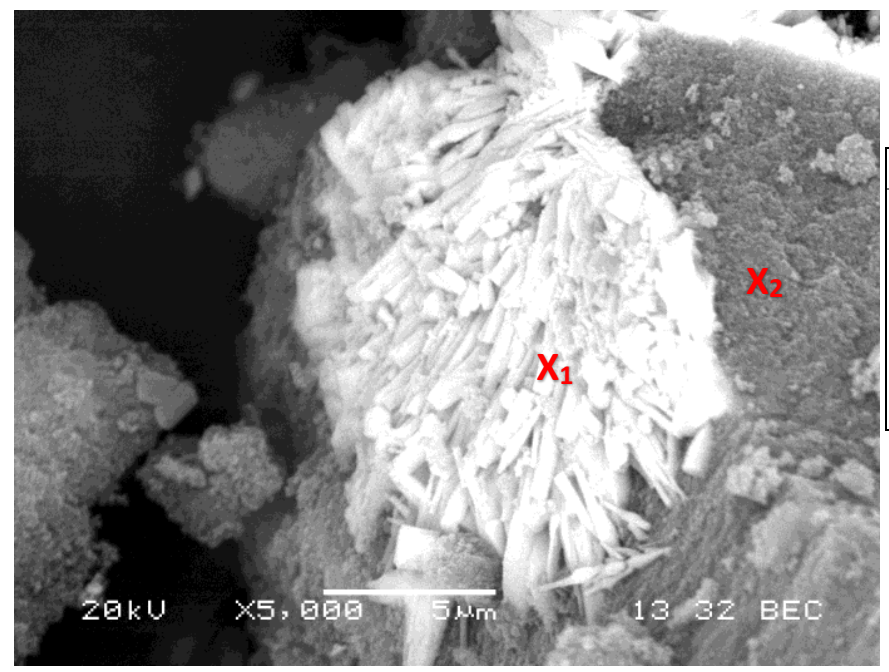

\begin{tabular}{|c|c|c|c|}
\hline \multicolumn{2}{|c|}{$\mathrm{X}_{1}$} & \multicolumn{2}{|c|}{$\mathrm{X}_{2}$} \\
\hline Element & At\% & Element & At \% \\
\hline $\mathbf{C}-\mathbf{K}_{\boldsymbol{\alpha}}$ & 26.58 & $C-K_{\alpha}$ & 0.00 \\
\hline $\mathbf{O}-\mathbf{K}_{\boldsymbol{\alpha}}$ & 50.99 & $\mathbf{O}-\mathbf{K}_{\boldsymbol{\alpha}}$ & 61.27 \\
\hline $\mathbf{N a}-\mathbf{K}_{\alpha}$ & 15.28 & $\mathbf{N a}-\mathbf{K}_{\boldsymbol{\alpha}}$ & 06.45 \\
\hline $\mathbf{S i}-\mathbf{K}_{\boldsymbol{\alpha}}$ & 04.81 & $\mathbf{A l}-\mathbf{K}_{\boldsymbol{\alpha}}$ & 01.60 \\
\hline $\mathbf{U}-\mathbf{M}_{\boldsymbol{\alpha}}$ & 01.77 & $\mathbf{S i}-\mathbf{K}_{\boldsymbol{\alpha}}$ & 28.06 \\
\hline $\mathbf{K}-\mathbf{K}_{\boldsymbol{\alpha}}$ & 00.57 & $\mathbf{U}-\mathbf{M}_{\boldsymbol{\alpha}}$ & 0.00 \\
\hline & & $\mathbf{K}-\mathbf{K}_{\boldsymbol{\alpha}}$ & 02.61 \\
\hline
\end{tabular}

Figure 13. SEM image and EDS data showing fragmented remains of needle-like structures in the ground $\mathrm{HBi}-\mathrm{HCa}-2 \mathrm{~W}$ sample

\subsection{Conclusions}

This preliminary investigation made use of an array of synthetic pore water solutions alongside the laboratory scale application of the ammonia gas injection method for remediation to attempt to mimic the potential uranium rich species that would be formed. The exploratory look at the process and its products were used to develop the direction of the overall study and further the attempt to characterize its products. 
The results of $\mathrm{x}$-ray diffraction were inconclusive in that no crystalline uranium phase was irrefutably identified using the comparison of the sample diffraction pattern and reference data. Though analysis of the precipitate was unclear regarding the uranium-phase, it was recognized that vast amounts of nitratine were produced in the experimental system. Considering the high concentrations of sodium and nitrate used in the synthetic pore water solution and the ammonia gas injection-induced nitrification of the aqueous solution, there are multiple pathways capable of producing the much observed nitratine salt. Despite its relatively high solubility, the precipitation of the salt is reasonable considering the saturated conditions of the synthetic pore water solution. It was speculated that the massive presence of nitratine could be stifling to the relatively scarce uranium-rich analyte. In order to reduce the potential effect of this overwhelming nitratine presence, the sample preparation method was modified to include a vacuum filtration and rinse step. The approach would also likely reduce the presence of water soluble uranyl-carbonate species like that which was tentatively identified as cejkaite in the high bicarbonate samples.

Despite the paucity of uranium-bearing species observed in the low-bicarbonate samples that resulted in the category's exclusion in the majority of these initial assessments, the low-bicarbonate samples were evaluated in further experiments because of the interest in their role in the speciation of uranium in the resultant products. Though the time study used to determine the development time that would result in the highest yield of the anticipated uranium-rich precipitate showed the 2-week period to be most productive, it was not used in subsequent sample preparation. Alternatively, the sample was open to air to allow the $\mathrm{pH}$ to independently drop and stabilize, similarly to what would be expected in the in-situ system it was intended to represent. 


\section{Optimization and Analysis of the Products of the $\mathrm{NH}_{3}$ Injection Method}

\subsection{Synthetic Pore Water System Optimization}

Initial laboratory scale experiments to investigate the ammonia gas injection remediation method were completed using an array of synthetic porewater solutions designed to represent the conditions of the Hanford 200 Area vadose zone. Analysis of the resultant precipitates using scanning electron microscopy with energy dispersive spectroscopy (SEM-EDS) analysis revealed the presence of a seemingly crystalline uranium-rich solid which would become the focus of characterization efforts. The comparison of sample diffraction patterns, obtained using powder X-ray diffraction (XRD) analysis, to reference patterns for the most likely mineral phases resulted in tentative matches for nitratine $\left(\mathrm{NaNO}_{3}\right)$ and cejkaite $\left(\mathrm{Na}_{4}\left(\mathrm{UO}_{2}\right)\left(\mathrm{CO}_{3}\right)_{3}\right)$. These findings were considered problematic because of the high solubility of both species. Nitratine in particular was found in such significant amounts that it was theorized that its overwhelming presence hindered the detection of less abundant species like the uranium-bearing precipitate of interest.

To address these complications, the preparation and evaluation of samples were modified to include filtration and rinse steps in order to lessen the interference of ancillary species that may precipitate in sample drying steps. These changes were directly implemented into the consequent efforts to optimize the synthetic pore water system to maximize the fraction of uranium in the precipitate phase and facilitate solid phase characterization. Additionally, the concentration of uranium used in synthetic porewater solution was more than doubled from $200 \mathrm{ppm}(0.84 \mathrm{mM})$ to $500 \mathrm{ppm}(\sim 2.1 \mathrm{mM})$; still within the range found in the

Hanford 200 Area vadose zone (Serne et al., 2008). The study used a $3^{2}$ full-factorial 
experimental design in order to cover all combinations of the two-factor, three-level experiment. The experiment would involved preparing synthetic pore water solutions with a range of concentrations for the experimental variables, bicarbonate $\left(\mathrm{HCO}_{3}^{-}\right)$and calcium $\left(\mathrm{Ca}^{2+}\right)$ resulting in a total of 9 samples before duplicates.

\subsubsection{Methods}

The modified sample preparation methods closely follow the main components of the procedure which were described in Initial Systems and Analysis. An array of synthetic pore water solutions was put together using stock solutions of their component salts, combined to achieve the desired final concentrations when diluted to volume (Table 4). Samples were prepared in duplicate in order to compare the observations with and without the DIW rinse added to the preparation procedure.

Table 4. Stock Solution \& Synthetic Pore Water Concentrations for Sample Preparation

\begin{tabular}{|c|c|c|}
\hline Stock Solution & Concentration $(\mathbf{m M})$ & $\begin{array}{c}\text { Synthetic Pore Water } \\
\text { Concentrations }(\mathbf{m M})\end{array}$ \\
\hline $\mathbf{C a C l}_{2} \cdot \mathbf{6 H}_{2} \mathbf{O}$ & 500.00 & $0 / 5 / 10$ \\
\hline $\mathbf{N a H C O}_{3}$ & 400.00 & $5 / 25 / 50$ \\
\hline $\mathbf{N a}_{2} \mathbf{S i O}_{3} \cdot \mathbf{9 H}_{2} \mathbf{O}$ & 422.24 & 100 \\
\hline $\mathbf{A l}_{\mathbf{N O}} \mathbf{N O}_{3} \cdot \mathbf{9 H}_{2} \mathbf{O}$ & 50.00 & 5 \\
\hline $\mathbf{U O}_{2}\left(\mathbf{N O}_{3}\right)_{2} \cdot \mathbf{6 H}_{2} \mathbf{O}$ & 210.06 & $2.1(500 \mathrm{ppm} \mathrm{U})$ \\
\hline $\mathbf{5 \%} \mathbf{N H}_{3}$ in $\mathbf{N}_{\mathbf{2}}$ (gas) & Bubbled into solution until pH $\approx 11$ \\
\hline
\end{tabular}

The procedure begins with concentrated stock solutions of $\mathrm{KHCO}_{3}, \mathrm{Na}_{2} \mathrm{SiO}_{3}$, and $\mathrm{Al}\left(\mathrm{NO}_{3}\right)_{3}$ prepared to form the base of the various synthetic pore water solutions. The base solution would then be $\mathrm{pH}$ adjusted using nitric acid to reach a value of about 8 , consistent with the pH of pore water in the Hanford vadose zone (Serne et al., 2008). The synthetic pore water solutions were then bubbled with ammonia gas until the system reached a treatment $\mathrm{pH}$ range around 11 (Figure 14). Immediately following this step, the base solutions were 
broken up into three equivalent aliquots, for each of the desired calcium concentrations. The final components, $\mathrm{CaCl}_{2}$ and $\mathrm{UO}_{2}\left(\mathrm{NO}_{3}\right)_{2}$, were added in small volumes of highly concentrated solutions in order to reach desired concentrations when diluted.

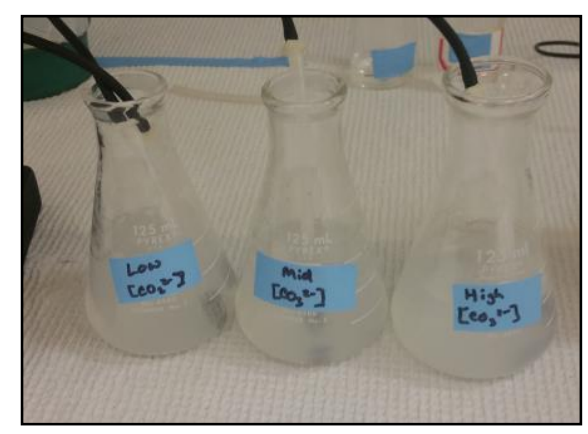

Figure 14. $\mathrm{NH}_{3}$ injection of the low, mid, and high bicarbonate synthetic pore water base solutions.

The synthetic pore water $\mathrm{pH}$ was monitored as the samples re-established the pre-treatment $\mathrm{pH}$ range through $\mathrm{CO}_{2}$ absorption and the partitioning and liberation of the dissolved $\mathrm{NH}_{3}$ gas. The change in $\mathrm{pH}$ was very slow initially, dropping by less than 0.5 in the first week. After three weeks of slow change, the samples were transferred to an orbital shaker and agitated gently for four additional weeks before reaching the desired post-treatment $\mathrm{pH}$ range of $8-9$. The solid precipitate phase and supernatant were separated by vacuum filtration using disposable $0.22 \mu \mathrm{m}$ nitrocellulose filters. The collected precipitates of sample duplicates, prepared identically to this point, were rinse with $5 \mathrm{~mL}$ of deionized water. The filtered precipitates were dried at $30{ }^{\circ} \mathrm{C}$ over three days while the supernatant filtrates and rinse solutions were labeled and stored for further analysis. 
Table 5. Synthetic Pore Water Solution Concentrations \& Labels

\begin{tabular}{|c|c|c|c|c|c|c|c|c|c|}
\hline \multirow{2}{*}{$\begin{array}{c}\text { Primary } \\
\text { Constituent } \\
\text { s }\end{array}$} & \multicolumn{9}{|c|}{ Component Concentrations } \\
\hline & \multicolumn{3}{|c|}{ Low Bicarbonate } & \multicolumn{3}{|c|}{ Mid Bicarbonate } & \multicolumn{3}{|c|}{ High Bicarbonate } \\
\hline $\mathrm{HCO}_{3}^{-}$ & \multicolumn{3}{|c|}{5} & \multicolumn{3}{|c|}{25} & \multicolumn{3}{|c|}{50} \\
\hline Al & \multirow{2}{*}{\multicolumn{3}{|c|}{$\begin{array}{c}5 \\
100\end{array}$}} & \multicolumn{3}{|c|}{5} & \multicolumn{3}{|c|}{5} \\
\hline Si & & & & \multicolumn{3}{|c|}{100} & \multicolumn{3}{|c|}{100} \\
\hline $\mathbf{C a}$ & 0 & 5 & 10 & 0 & 5 & 10 & 0 & 5 & 10 \\
\hline Label & $05-00$ & $05-05$ & $05-10$ & $25-00$ & $25-05$ & $25-10$ & $50-00$ & $50-05$ & $50-10$ \\
\hline
\end{tabular}

Samples were labeled using the two digits of the bicarbonate concentration followed by the two digits of the calcium concentration (Table 5). Either an A or B was affixed to the end of the label to distinguish the unrinsed and rinsed samples, respectively. The concentrations of uranium retained in the supernatant filtrate solution were used to inferred which samples precipitated the most uranium. This included the assumption of negligible analyte loss to the filter. Supernatant solutions were diluted up to 10,000 times in $1 \%$ nitric acid to ensure that that analyte fell into the analytical range for analysis by kinetic phosphorescence analyzer (KPA). The results of this analysis were used a primary basis for paring down the number of samples advanced to further analysis.

\subsubsection{Results \& Discussion}

The results of previous attempts to characterize the precipitates produced by application of the remediation method to synthetic pore water solutions showed that solid phase analysis was largely hindered by the relatively small amount of uranium analyte in the precipitate. To counter the low abundance of uranium analyte, the present optimization study focused on what component concentrations would maximize the fraction or uranium in the precipitate phase determined by the concentrations of uranium left in their supernatants. The method relied on the assumption that all uranium introduced to the sample solutions was either retained in solution or precipitated/adsorbed onto the solid phase. The 
experiments were designed such that the results of the KPA analysis of the filtered supernatant solutions could be visualized using response surface diagrams (Figure 15). The full factorial experimental design considered all test concentrations to display the relationship between the two variable concentrations and the concentration of uranium in the supernatant phase. Though the duplicate sample set was rinsed after vacuum filtration, the data used in the response surface do not consider that rinse solution.

The results of both sample sets present a clear positive correlation between the increasing concentration of bicarbonate in synthetic porewater solutions and the concentration of uranium in the filtered post-treatment supernatant solution. The finding suggests that with increasing sample bicarbonate concentration, the amount of uranium in the precipitate decreases. It is therefore safe to conclude that the high bicarbonate samples (50-00, 50-05, $\& 50-10$ ) would be least likely to precipitate the uranium analyte.
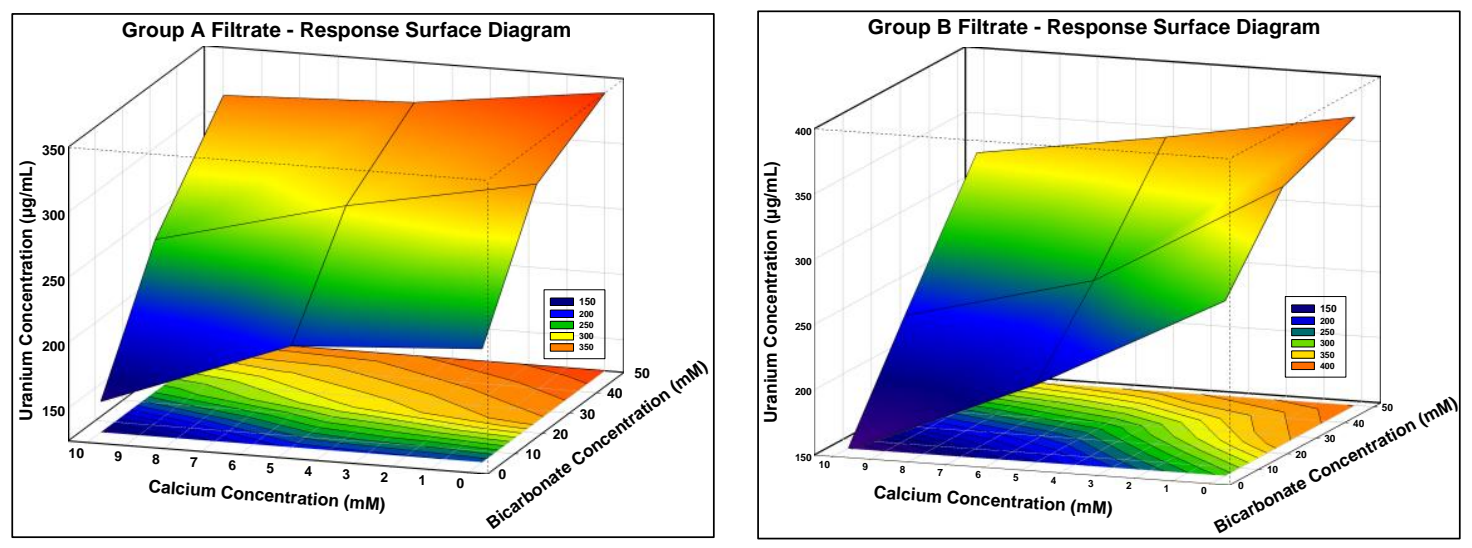

Figure 15. Response surface diagrams displaying filtrate solution uranium retention for the original (Group A) and duplicate/rinsed (Group B) samples

The observed trend of uranium in the supernatant solutions increasing with added bicarbonate is likely indicative of conditions increasingly favoring the formation of uranyl carbonate. These species, which are highly soluble in aqueous solutions, form charged 
complexes which can adsorb to the surface solid minerals under the right $\mathrm{pH}$ conditions. These data conflict with the results of scanning electron microscope analysis for the initial samples, described in the Initial Systems and Analysis section, where uranium-rich crystalline solid phases were exclusive to the high bicarbonate $(50 \mathrm{mM})$ samples. Within that same sample set, no samples showed these crystalline structures, though a scarce few did show amorphous uranium phases observable by SEM analysis. The SEM analysis results run counter to what is expected of low bicarbonate samples depending on the response surface. The trends in Figure 15 show that low bicarbonate samples (05-00, 05$25, \& 05-50$ ) have the least uranium remaining in the supernatant solutions and should, therefore, have the most in the precipitate phase.

Though much less pronounced, there is correlation between the increasing calcium concentrations in sample solution and the concentration of uranium in the supernatant. Unlike bicarbonate, however, the increasing calcium is associated with a decrease of uranium concentration in solution and, therefore, a reciprocal increase in the uranium precipitated out. It was posited that the increase in calcium could favor the removal or uranium in one of two ways. There is evidence, gathered from speciation modeling, to suggest increasing the calcium in the system could facilitate the formation of calciumuranyl precipitate phases (Lagos et al., 2016). Another possibility is that the increase in calcium results in the formation of more stable calcium-uranyl-carbonate species or the precipitation of solids, such as calcium carbonate or calcium silicates, which could serve as nucleation sites provoking silica polymerization reactions and the precipitation of silica (Iler, 1979) along with the adsorption or co-precipitation of uranium species. 
Despite the initial aim of simply determining which sample conditions most favored the partitioning of uranium from the sample solutions to the resulting precipitates, all samples continued on to the following precipitate analysis. In the continued analysis, particular attention was paid to the samples corresponding to the vertices of the response surface diagrams $(05-00,05-10,50-00, \& 50-10)$.

\subsection{Characterization of the Uranium-Bearing Solid Phase}

The analysis and characterization of the uranium-bearing solids made use of the precipitates prepared in the preceding optimization study. The analysis began with the imaging of the sample surface using SEM with EDS to identify areas of higher than average

uranium content, similar to what was described in the initial investigation and previously conveyed in published reports (Lagos et al., 2013; Lagos et al., 2014). From there, a sequential extraction procedure was employed to attempt to classify the predominant uranium species being produced depending on its interactions with various classes of solutions. In the procedure samples are treated with a series of increasingly aggressive extraction solutions and conditions, each selected to target particular, increasingly difficult to remove uranium phases.

Perhaps most notably implemented for sediment extraction of trace metals by Tessier et al. (Tessier, Campbell, \& Bisson, 1979), the sequential extraction method has been adjusted and applied to various elements and sediment systems over time. These include several targeting the fractionation of heavy metals (i.e., $\mathrm{Cd}, \mathrm{Cr}, \mathrm{Pb}$, and $\mathrm{Zn}$ ) and actinides (i.e., $\mathrm{Am}$, $\mathrm{Pu}$, and $\mathrm{U}$ ) from reference and natural solid and sediment samples (Kohler, Curtis, Meece, \& Davis, 2004; Rauret et al., 1999; Schultz, Burnett, \& Inn, 1998; Smith \& Szecsody, 
2011). The present study employed a sequential extraction procedure modified for application to the precipitate sample but following primarily the methods reported by Smith \& Szecsody et al., in association with Pacific Northwest National Laboratory, who also provided support for this research.

Though fundamentally similar to SEM-EDS analysis, the electron probe microanalyzer (EPMA) capability for fine analysis and high spatial resolution is unmatched by the former technique. Limited access to the instrument and the sample preparation requirements, including being shipped to and from collaborators at Pacific Northwest National Laboratory, demanded that samples be limited to those which could provide the most insight. Those samples, the selection of which was determined by the findings of the preceding analyses, were mapped by EPMA for the distribution of several key elements. The maps could be used to detail the elemental associations of uranium to support the findings of other analyses.

\subsubsection{Methods}

\section{SEM-EDS Analysis}

Sample specimens were taken from all precipitates produced in the preceding optimization study for solid phases analysis by scanning electron microscopy with energy dispersive spectroscopy (SEM-EDS). The SEM-EDS analysis was designed to, together with the results of the synthetic pore water optimization study, determine what samples would move on to further analysis.

Samples were mounted to aluminum studs using double-sided carbon tape and sputter coated with a thin layer of gold using an SPI-Module Control and Sputter unit to enhance 
surface conductivity for better imaging. Analysis was done using a JEOL-5910-LV SEM with acceleration potentials between 10 and $20 \mathrm{kV}$. The EDS analysis was done using an EDAX Sapphire detector with UTW Window and Genesis EDS microanalysis software. Micrographs were completed primarily using backscatter electron capture mode in order to better discern the areas with higher average atomic mass, which would be most likely to be rich in uranium.

\section{Sequential Extraction}

The sequential extraction procedure described in my study is primarily followed the methods published by Smith \& Szecsody for use with sediment from the Hanford 300 and 200 areas (Smith \& Szecsody, 2011; Szecsody et al., 2012). The principal differences from the source methods were the choice of distilled deionized water (DDIW) for the initial extraction (Step I), rather than a synthetic ground water solution, and the omission of the oxalate extraction which preceded the terminal nitric acid extraction (Table 6). The latter was omitted because it was meant to target iron oxides from soils, which were not relevant to the system covered by my study. Additionally, after each extraction step samples were rinsed with $5 \mathrm{~mL}$ of deionized water (DIW) in order to minimize incidental transfer or residual leachate to the next step. For analytical purposes, this rinse solution was considered a part of the preceding extraction. 
Table 6. Sequential Extraction Procedure

\begin{tabular}{|c|c|c|c|c|}
\hline $\begin{array}{l}\text { Extraction } \\
\text { Step }\end{array}$ & $\begin{array}{l}\text { Extraction } \\
\text { Solution }\end{array}$ & Concentrations/Notes & $\begin{array}{l}\text { Extraction } \\
\text { Time }\end{array}$ & Target \\
\hline I & DDI-Water & - & 1 hour & Aqueous species \\
\hline II & $\begin{array}{l}\text { Carbonate } \\
\text { Buffer }\end{array}$ & $\begin{array}{c}0.0144 \mathrm{M} \mathrm{NaHCO} \\
\mathrm{Na}_{2} \mathrm{CO}_{3} \text {, pH } 9.3\end{array}$ & 1 hour & Adsorbed species \\
\hline III & $\begin{array}{l}\text { Acetate } \\
\text { Buffer }\end{array}$ & $\begin{array}{c}1 \mathrm{M} \mathrm{CH} \mathrm{CH}_{3} \mathrm{COO}-\mathrm{Na} \\
\text { (Adjusted to } \mathrm{pH} 5 \text { by Acetic Acid) }\end{array}$ & 1 hour & Some carbonates \\
\hline IV & Acetic Acid & Concentrated $\mathrm{CH}_{3} \mathrm{COOH}, \mathrm{pH} 2.3$ & 5 days & $\begin{array}{c}\text { Carbonates and } \\
\text { hydrated silicates }\end{array}$ \\
\hline $\mathbf{V}$ & Nitric Acid & $8 \mathrm{M} \mathrm{HNO}_{3}$, at $95^{\circ} \mathrm{C}$ & 2 hours & $\begin{array}{l}\text { Difficult to remove } \\
\text { phases }\end{array}$ \\
\hline
\end{tabular}

Like other aspects of the procedure, a variety of methods for selecting the volume of extraction solution has been published (Ariza, Giraldez, Sanchez-Rodas, \& Morales, 2000; Galán et al., 2003; Tessier et al., 1979). These range from using a consistent volume to applying any of a number to solid-to-solution ratios. For the purpose of my study, the extraction volume was selected using a 40-to-1 solid $(\mathrm{mg})$ to solution $(\mathrm{mL})$ ratio which was used in a similar PNNL extraction study on uranium in Hanford sediment (Smith \& Szecsody, 2011).

Table 7. Extraction Solution Volumes - by Sample

\begin{tabular}{|c|c|c|}
\hline $\begin{array}{c}\text { Sample } \\
\text { Label }\end{array}$ & Sample Weight $(\mathbf{m g})$ & $\begin{array}{c}\mathbf{4 0 : 1} \text { Extraction Volume } \\
(\mathbf{m L})\end{array}$ \\
\hline $\mathbf{0 5}-\mathbf{0 0 A}$ & 26.4 & 0.6600 \\
\hline $\mathbf{0 5}-\mathbf{0 0 B}$ & 16.6 & 0.4150 \\
\hline $\mathbf{0 5}-\mathbf{1 0 A}$ & 38.2 & 0.9550 \\
\hline $\mathbf{0 5}-\mathbf{1 0 B}$ & 45.8 & 1.145 \\
\hline $\mathbf{5 0}-\mathbf{0 0 A}$ & 25.0 & 0.6250 \\
\hline $\mathbf{5 0}-\mathbf{0 0 B}$ & 19.1 & 0.4775 \\
\hline $\mathbf{5 0}-\mathbf{1 0 A}$ & 32.1 & 0.8025 \\
\hline $\mathbf{5 0}-\mathbf{1 0 B}$ & 39.4 & 0.9850 \\
\hline
\end{tabular}

The extraction procedure began with the addition of a known mass of precipitate to a labeled vial, to which the corresponding volume of extraction solution would be added (Table 7). The mixture was briefly vortexed before being transferred to an orbital shaker where the vessel was agitated at $150 \mathrm{rpm}$ for the duration of the extraction. After extraction, 
samples were centrifuged at $5000 \mathrm{rpm}$ for 30 minutes in order to separate the extractant and remaining precipitate. The extraction was followed by a 10-minute DI water rinse which, like the extraction, was accompanied with agitation and centrifugation. The process of extraction and rinse was repeated for extraction steps I through IV with each of their specified extraction times. The final extraction (Step V), intended to target hard to extract uranium species, differed in that its extraction solution used $8 \mathrm{M}$ nitric acid $\left(\mathrm{HNO}_{3}\right)$ maintained at $95^{\circ} \mathrm{C}$ using a water bath. Sample rinses and leachates were collected and diluted $10-10,000$ times for KPA analysis to determine uranium concentrations.

\section{Electron Probe Microanalysis}

The EPMA elemental mapping was limited to the unrinsed samples associated with the vertices of the surface response diagrams described in 4.1 Synthetic Pore Water System Optimization. Using these results, the low bicarbonate samples (05-00A \& 05-10A) showed the least uranium retained in the supernatant solution and therefore should have had the most in the precipitate. Additionally, the high bicarbonate (50-00A \& 50-10A) precipitates were selected using the fact that similar high bicarbonate samples have regularly shown uranium-rich precipitates in SEM-EDS analysis (Figure $6 \&$ Figure 7).

Specimens from each of the selected samples were crushed and mixed into Beuhler EpoThin 2, a clear low viscosity epoxy capable of curing at room temperature. The suspension was poured into a $1 / 4$ " hole drilled into the centers of 1 -inch cylindrical epoxy molds, prepared beforehand, in order to concentrate the analysis area (Figure 16). The samples spent 5 minutes in a vacuum chamber at $25 \mathrm{in}$. $\mathrm{Hg}$ to evacuate any air bubbles before being allowed to cure for 24 hours. The samples were then shipped to PNNL 
collaborators for cutting, grinding, and polishing steps, which required instruments and facilities dedicated to samples containing radionuclides which were not locally available.

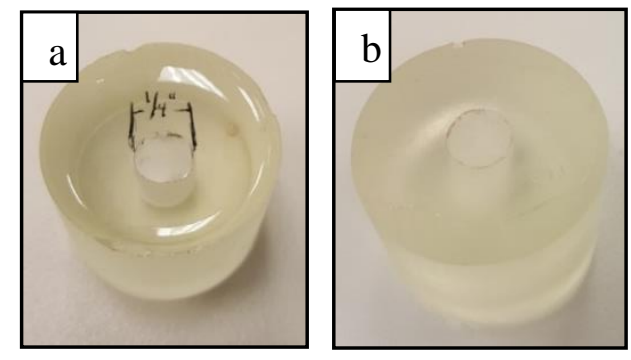

Figure 16. Epoxy molds before (a) and after (b) filling with resin + sample mixtures.

Once returned, the polished samples were mapped using EPMA's high spatial resolution elemental analysis. A JEOL 8900R Superprobe equipped with 5 two-crystal WDS spectrometers and a single EDS-UTW detector was used to simultaneously detect multiple elements as the beam rastered across the sample surface. The instrument was run using a $20.0 \mathrm{kV}$ accelerating voltage, 5-10 micron spot size, and a $20 \mathrm{~ms}$ dwell time. For the majority of samples, an accumulation of 5 scans were used to create a comprehensive map for each targeted element.

\subsubsection{Results \& Discussion}

\section{SEM-EDS Analysis}

The SEM-EDS analysis of past samples played an important part in the evaluation these precipitates, including providing an idea of how to visually distinguish areas likely to have the highest atomic percentage of uranium. Primary areas of interest included crystal-like structures and areas of higher average atomic mass, identified as bright spots using backscatter electron capture mode. Energy dispersive spectroscopy was used to confirm 
the presence and estimate the abundance of uranium in these analysis points using primarily the atomic percentage (At\%).

Surface morphological and compositional analysis started with low bicarbonate samples because of the relatively high removal of uranium from sample solutions and, presumably, into the precipitate which was observed in supernatant analysis. The observed trends strongly imply that these precipitates would have the most abundant uranium phases for identification by SEM-EDS analysis. To the contrary, of the low bicarbonate samples across the spectrum of calcium concentrations, both unrinsed (Figure 17) and rinsed (Figure 18), no point analysis showed atomic percentages of uranium reaching or exceeding even $1 \%$. The value is one that was regularly exceeded in similar samples, where uranium-phases regularly reached $1-5 \%$. 


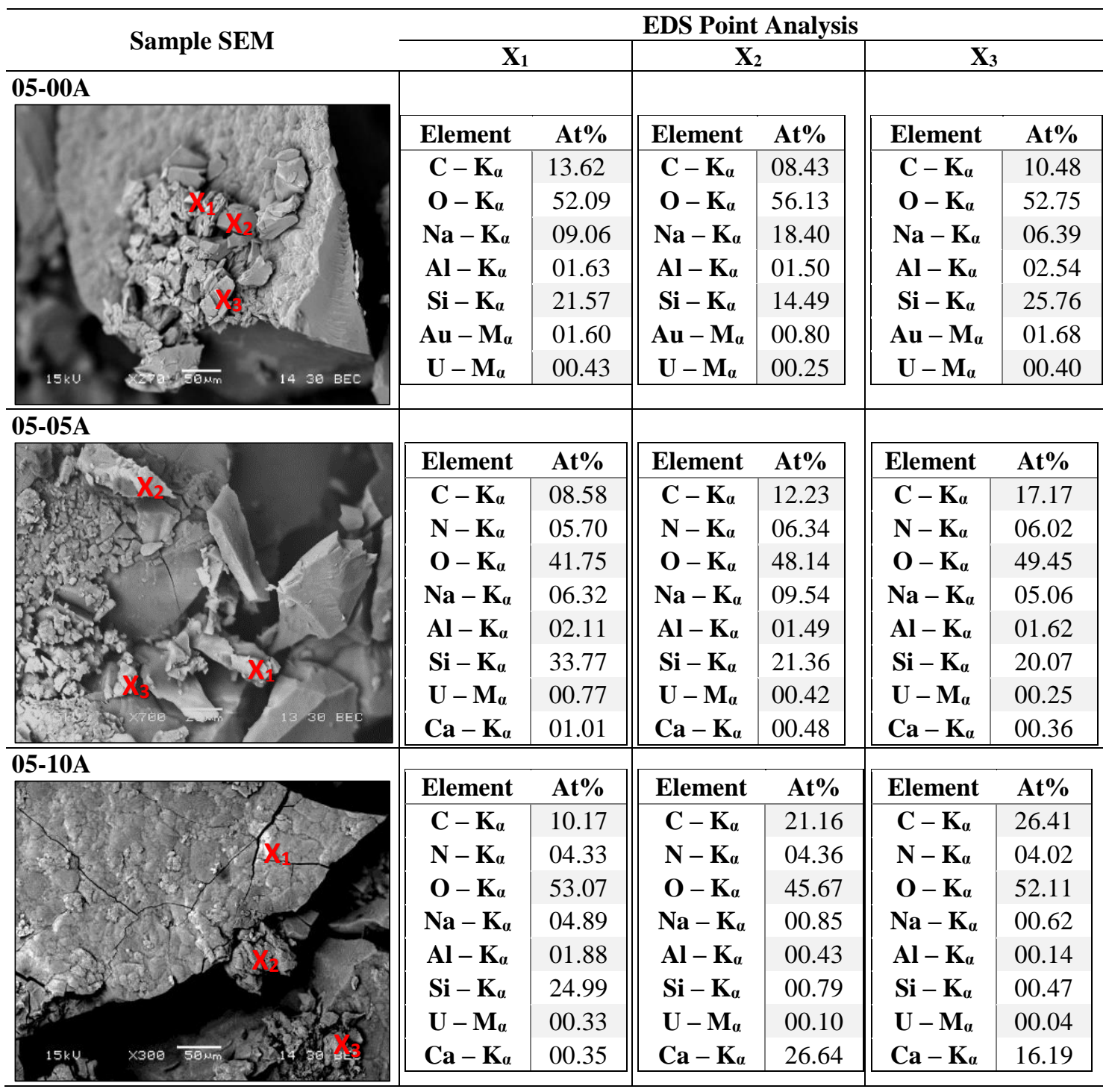

Figure 17. SEM images and EDS data for unrinsed low bicarbonate $(5 \mathrm{mM})$ samples

There are several possible explanations for the discrepancy between the expectation of finding uranium-rich phases and the lack there of in solid phase analysis. The simplest reasoning is that the sample specimens extracted for SEM-EDS analysis did not contain any of the uranium phases present in the sample. More likely though is that the uranium phases present were either significantly coated by re-precipitated phases or highly distributed throughout the sample and indistinguishable by backscatter SEM. Though the low bicarbonate samples showed none of the uranium-rich regions observed in initial 
analysis, there is a trend with the most uranium-abundant analysis points $(\mathrm{At} \% \geq 0.5)$ also having the highest abundance of silicon (i.e., 05-05A- $\mathrm{X}_{1}, 05-00 \mathrm{~B}-\mathrm{X}_{1}, 05-10 \mathrm{~B}-\mathrm{X}_{1}$, and 05$\left.10 \mathrm{~B}-\mathrm{X}_{3}\right)$ which could suggest an association consistent with the formation of uranylsilicates or the coating of uranium with silica species (Figure $17 \&$ Figure 18).

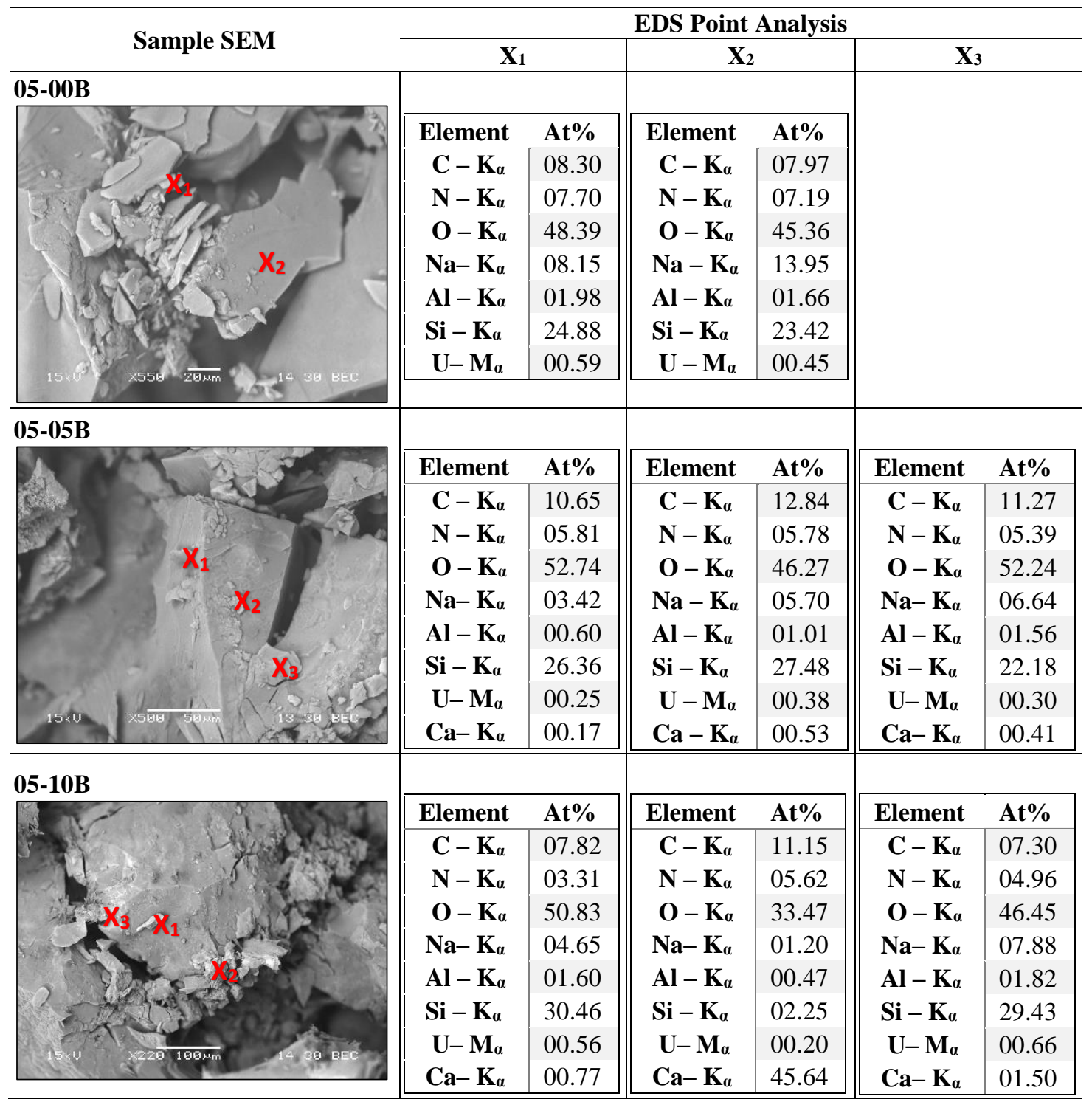

Figure 18. SEM images and EDS data for rinsed low bicarbonate $(5 \mathrm{mM})$ samples

The SEM imaging and EDS analysis (not shown) of the mid-range $25 \mathrm{mM}$ samples were largely the same across the range of calcium concentrations with a similar lack of uranium- 
rich areas of interest with exceptions in the 25-00B and 25-05A samples (Figure 19) which were the only ones to show uranium atomic percentages that exceeded $1 \%$. These areas of interest were different from past uranium-rich locations in that they appeared as flat, dark areas rather than the bright, crystal-like phases observed in past samples (Figure 6 \& Figure 7). The observed deviation is likely related to the increased silicon, the percentage of which is twice that of the EDS analysis of uranium phases typically spotted on similar samples. Like the aforementioned low bicarbonate samples, these regions of higher uranium and silicon abundance are likely the result of either uranyl-silicate formation or the coating of uranium species. The lack of significant brightness in the backscattered image, associated with areas of higher average atomic number, suggest that the latter is more likely.

25-00B

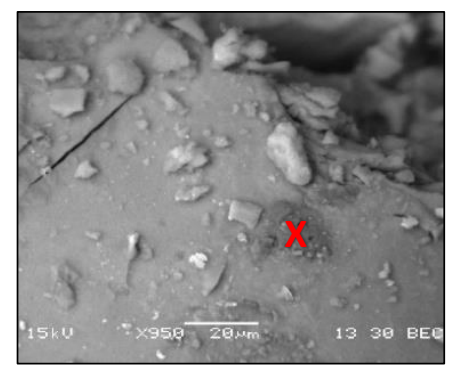

\begin{tabular}{|c|c|}
\hline Element & $\mathbf{A t} \%$ \\
\hline $\mathbf{C}-\mathbf{K}_{\boldsymbol{a}}$ & 03.17 \\
$\mathbf{N}-\mathbf{K}_{\boldsymbol{a}}$ & 13.94 \\
$\mathbf{O}-\mathbf{K}_{\boldsymbol{\alpha}}$ & 33.25 \\
$\mathbf{N a}-\mathbf{K}_{\boldsymbol{a}}$ & 11.96 \\
$\mathbf{A l}-\mathbf{K}_{\boldsymbol{\alpha}}$ & 01.77 \\
$\mathbf{S i}-\mathbf{K}_{\boldsymbol{\alpha}}$ & 34.90 \\
$\mathbf{U}-\mathbf{M}_{\boldsymbol{\alpha}}$ & 01.01 \\
\hline
\end{tabular}

25-05A

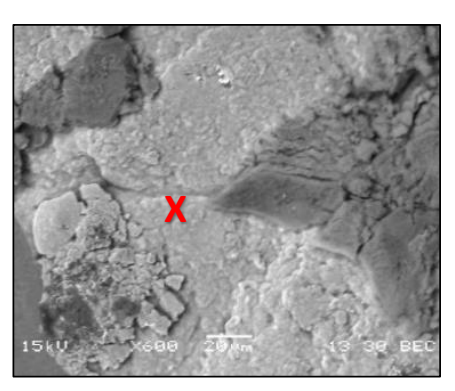

\begin{tabular}{|r|r|}
\hline Element & \multicolumn{1}{|c|}{$\mathbf{A t} \%$} \\
\hline $\mathbf{C}-\mathbf{K}_{\boldsymbol{\alpha}}$ & 04.64 \\
$\mathbf{N}-\mathbf{K}_{\boldsymbol{\alpha}}$ & 10.27 \\
$\mathbf{O}-\mathbf{K}_{\boldsymbol{\alpha}}$ & 32.18 \\
$\mathbf{N a}-\mathbf{K}_{\boldsymbol{\alpha}}$ & 08.77 \\
$\mathbf{A l}-\mathbf{K}_{\boldsymbol{\alpha}}$ & 02.14 \\
$\mathbf{S i}-\mathbf{K}_{\boldsymbol{\alpha}}$ & 39.27 \\
$\mathbf{U}-\mathbf{M}_{\boldsymbol{\alpha}}$ & 01.66 \\
$\mathbf{C a}-\mathbf{K}_{\boldsymbol{\alpha}}$ & 01.08 \\
\hline
\end{tabular}

Figure 19. SEM image and EDS data for point analysis of specimens from the rinsed, $25 \mathrm{mM}$ bicarbonate, zero calcium precipitate (left) and the unrinsed, $25 \mathrm{mM}$ bicarbonate, $5 \mathrm{mM}$ calcium precipitate (right)

50-00A

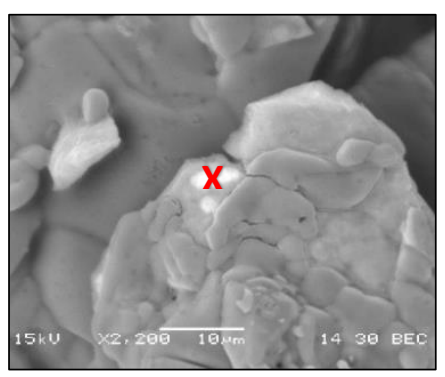

\begin{tabular}{|c|c|}
\hline Element & At\% \\
\hline $\mathbf{C}-\mathbf{K}_{\boldsymbol{\alpha}}$ & 11.22 \\
$\mathbf{N}-\mathbf{K}_{\boldsymbol{\alpha}}$ & 05.48 \\
$\mathbf{O}-\mathbf{K}_{\boldsymbol{\alpha}}$ & 55.08 \\
$\mathbf{N a}-\mathbf{K}_{\boldsymbol{\alpha}}$ & 06.11 \\
$\mathbf{A l}-\mathbf{K}_{\boldsymbol{\alpha}}$ & 01.40 \\
$\mathbf{S i}-\mathbf{K}_{\boldsymbol{\alpha}}$ & 19.38 \\
$\mathbf{U}-\mathbf{M}_{\boldsymbol{\alpha}}$ & 01.31 \\
\hline
\end{tabular}

50-05A

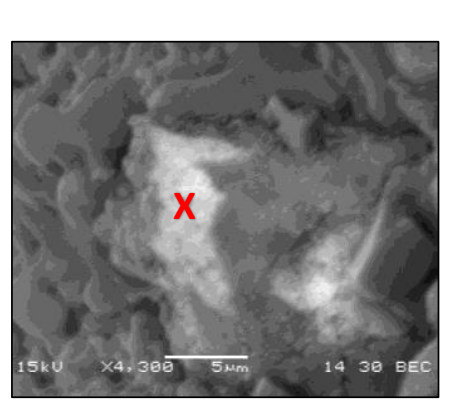

\begin{tabular}{|c|c|}
\hline Element & \multicolumn{1}{|c|}{ At\% } \\
\hline $\mathbf{C}-\mathbf{K}_{\boldsymbol{\alpha}}$ & 12.37 \\
$\mathbf{N}-\mathbf{K}_{\boldsymbol{\alpha}}$ & 07.13 \\
$\mathbf{O}-\mathbf{K}_{\boldsymbol{\alpha}}$ & 52.44 \\
$\mathbf{N a}-\mathbf{K}_{\boldsymbol{\alpha}}$ & 10.22 \\
$\mathbf{A l}-\mathbf{K}_{\boldsymbol{\alpha}}$ & 01.26 \\
$\mathbf{S i}-\mathbf{K}_{\boldsymbol{\alpha}}$ & 14.48 \\
$\mathbf{U}-\mathbf{M}_{\boldsymbol{\alpha}}$ & 01.47 \\
$\mathbf{C a}-\mathbf{K}_{\boldsymbol{a}}$ & 00.06 \\
$\mathbf{C l}-\mathbf{K}_{\boldsymbol{\alpha}}$ & 00.55 \\
\hline
\end{tabular}

Figure 20. SEM image and EDS data for point analysis of specimens from the unrinsed, $50 \mathrm{mM}$ bicarbonate, zero calcium precipitate (left) and the uninsed, $50 \mathrm{mM}$ bicarbonate, $5 \mathrm{mM}$ calcium precipitate (right) 
Although the overall trend in the optimization study suggested that uranium's partitioning into the precipitate would be favored in low bicarbonate samples, SEM-EDS analysis showed the distinct, bright, uranium-rich hotspots were exclusive to the high bicarbonate (50 mM) samples (Figure 20). These uranium phases, though they lacked the structure observed in prior samples, were consistent with those observed in HBi-LCa-3M (Figure 6) and $\mathrm{HBi}-\mathrm{HCa}-3 \mathrm{M}$ (Figure 7). The higher than average carbon and oxygen at these analysis points likely indicate the formation of a uranyl-carbonate species. It is possible that, despite the preventative addition of the vacuum filtration step, the evaporation of residual supernatant solution resulted in the re-precipitation or adsorption of otherwise soluble uranium species.

\section{Sequential Extraction Analysis}

The combination of supernatant analysis in the optimization study and SEM-EDS analysis of the sample precipitates was used to determine which samples would move on to the more extensive sequential extraction procedure. Though supernatant analysis suggested that uranium in the precipitate phase would be maximized in the low bicarbonate samples, SEM-EDS analysis primarily revealed uranium-rich phases in the high bicarbonate samples. While calcium concentration did appear to have an impact on uranium removal in the optimization study, the effect was much less pronounced than that of bicarbonate concentration. On the basis of these observations, specimens from all low bicarbonate $(5 \mathrm{mM})$ and high bicarbonate $(50 \mathrm{mM})$ samples were taken for the sequential extraction study. 
The KPA data collected from the study were plotted to display the mass of uranium removed with each extraction step using the calculated uranium concentration and the volume that it was extracted into (Figure 21). Despite the disagreement with the preceding SEM-EDS observations, the results of the sequential extraction were consistent with expectations using the supernatant analysis. Although its SEM-EDS analysis showed no observable uranium-rich phases, the total mass of uranium extracted shows that each of the low bicarbonate samples had more uranium removed than their high bicarbonate counterparts. The reduction in total mass uranium removed from solution could be explained by the formation of soluble uranyl-carbonates in solution that never made it through initial filtration and rinse steps. The difference is especially pronounced in the high calcium samples, in agreement with the results of with the supernatant analysis, which suggest that these would have the most uranium partitioning into the solid phase. In fact, there is a marked increase in total uranium removal in all calcium containing samples, compared to their calcium-free counterparts. Like the bicarbonate trend, this is consistent with the trend observed in the supernatant analysis in the synthetic porewater optimization study (Figure 15). In particular, the increase in total removal by the carbonate extraction solution, targeting adsorbed species, could indicate the formation of more stable calciumuranyl-carbonate species. 


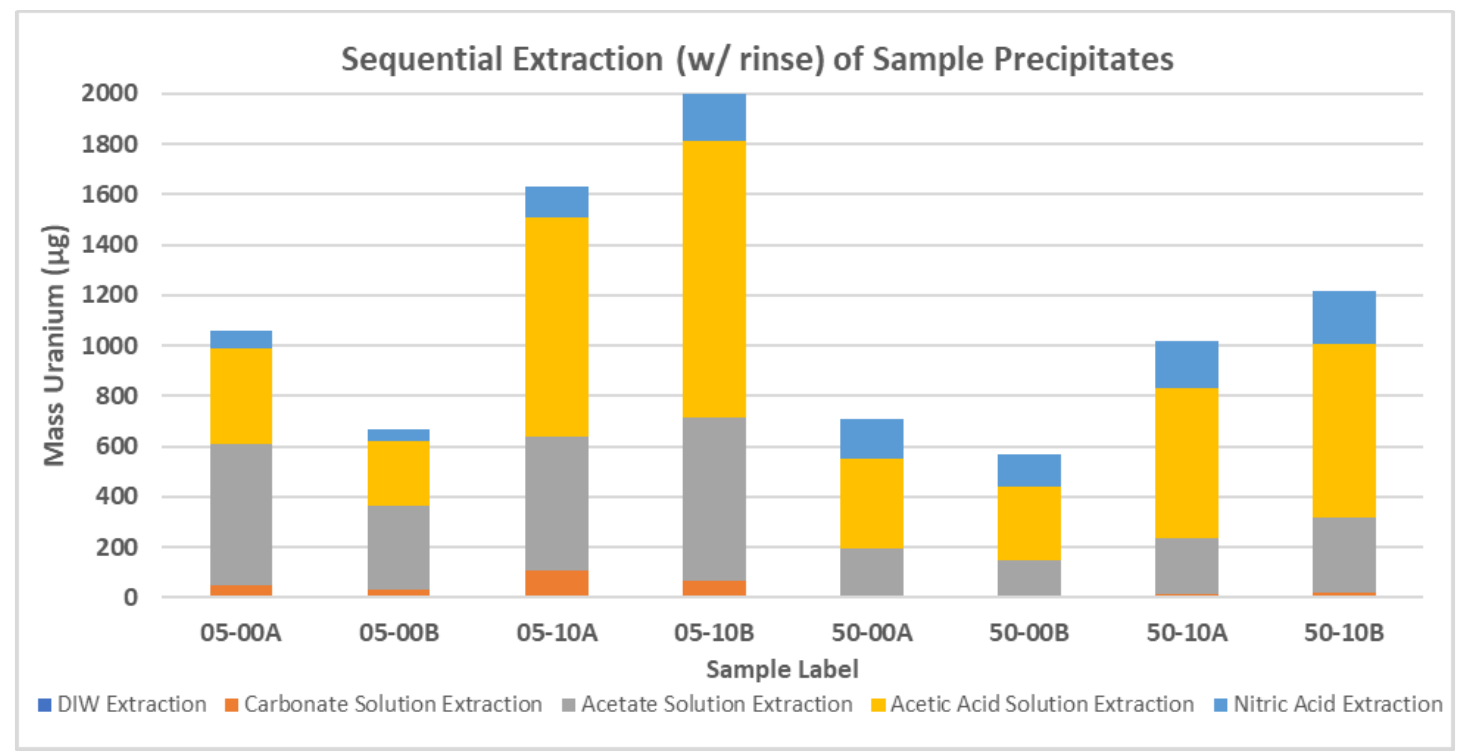

Figure 21. Sequential uranium extraction of sample precipitates (including rinse).

The comparison of the relative removal of uranium between the various extraction steps allows for developing associations using the preferential removal of target uranium phases (Table 6). The extraction distribution charts make clear that in both the rinsed (Figure 22) and unrinsed (Figure 23) samples, the DIW extraction (Step I) is the least effective. A miniscule mass removal in the DIW extraction suggests that the precipitates did not contain very much water-soluble uranium species. This would bode well for the goal of the remediation method, to lessen the mobility of uranium in the Hanford subsurface, but for the fact that the DIW extraction is not an ideal representative for the rain and groundwater that would most likely be interacting with the vadose zone contamination. This extraction could also have been affected by the fact that water soluble species were also specifically avoided with the changes to the sample preparation method (i.e.: vacuum filtration and rinsing). 


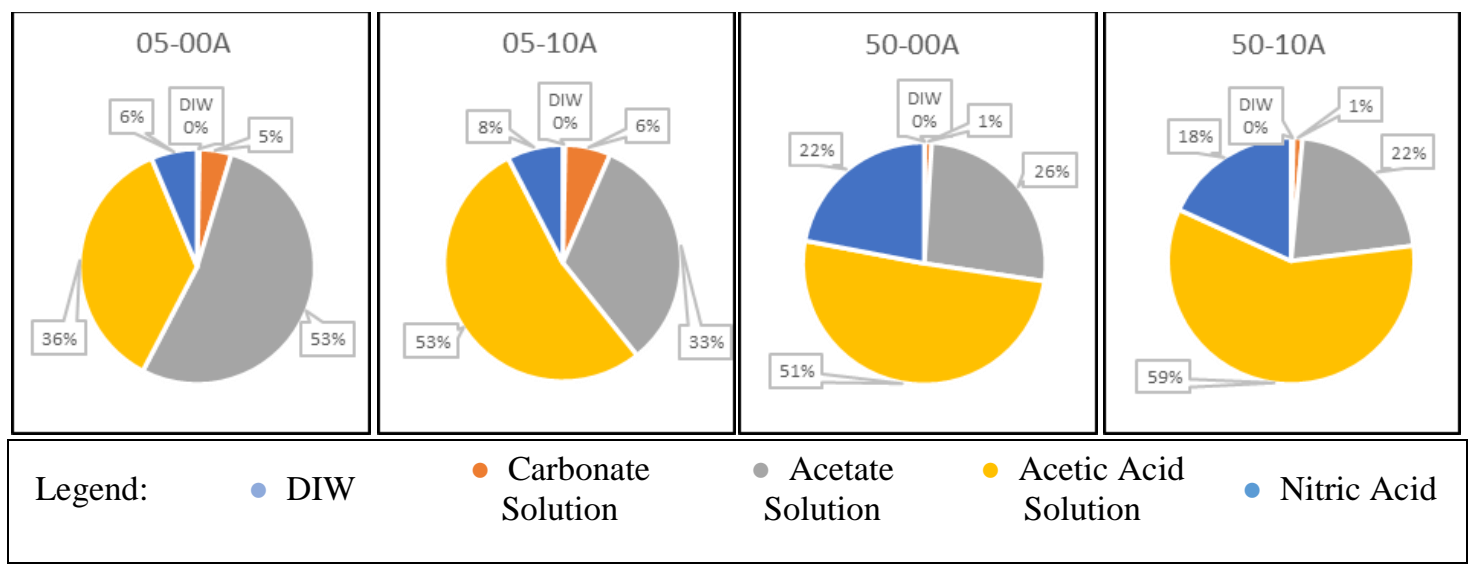

Figure 22. Uranium Extraction Distribution for Unrinsed (Group A) Samples.

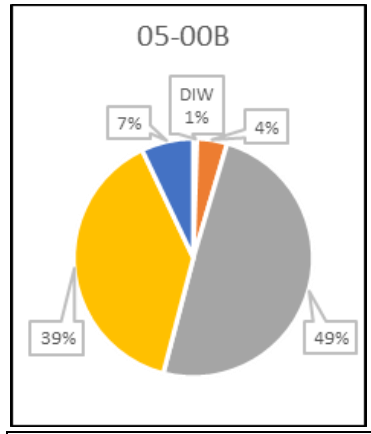

Legend:

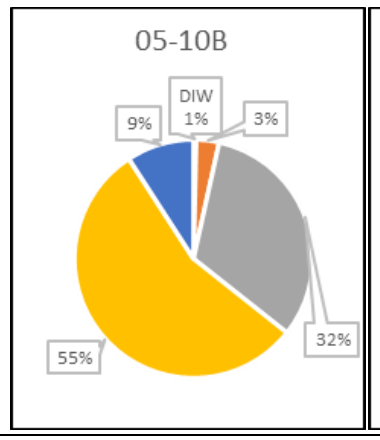

- Carbonate Solution

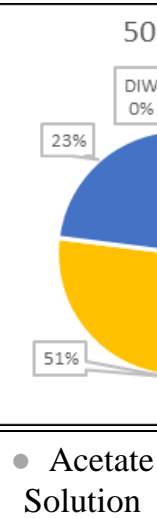

Solution
$50-00 B$

DIW
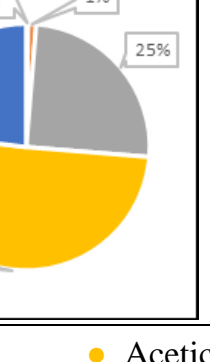

Solution

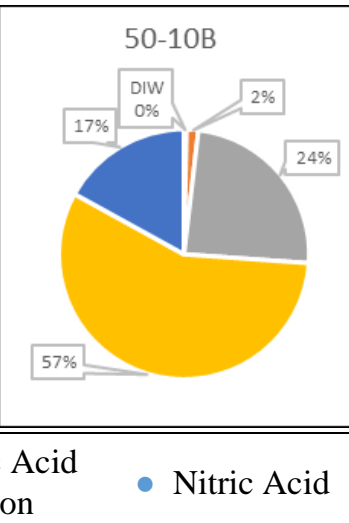

Figure 23. Uranium Extraction Distribution for Rinsed (Group B) Samples.

Between equivalent low and high bicarbonate samples, the carbonate extraction, which targets the adsorbed species, had a significant decrease in potency. In unrinsed samples, the relative uranium removal decreased from $5-6 \%$ to $1 \%$, about an $80 \%$ decrease of its relative abundance. A similar, less potent decline is observed in the rinsed samples. This change is likely indicative of a transition of the adsorbed uranyl species to a more difficult to remove phase with the increase in bicarbonate. The reduction in the extraction of aqueous species could be tied to the concurring increase in analyte extracted via the nitric acid extraction (Step V). In all corresponding high-low bicarbonate sample pairs, this extraction, which is used last to target the difficult to extract uranium phases, sees a significant increase where their share of the analyte distribution is doubled in high calcium 
samples, from $8-9 \%$ to $17-18 \%$, and more than tripled in low calcium samples, from 6-7\% to $22-23 \%$. Alternatively, this carbonate reduction could also be related to an increase in the acetic acid extraction, one of the more aggressive steps which targets the carbonates and hydrated silicates. The extraction saw a $12-15 \%$ increase in low calcium samples and a less compelling 2-6\% increase in high calcium samples.

The decrease in carbonate extraction corresponds only to a small portion of total increase in the acetic acid and nitric acid extractions. The rest of that increase comes from a corresponding decrease in the acetate extraction, the solution designated for the targeted removal of carbonates. The low bicarbonate - low calcium samples $(05-00 \mathrm{~A} / \mathrm{B})$ were the only samples to have acetate as the major extraction step; acetic acid led for all others. Of all sample sets, those extractions saw the most significant decrease between their low and high bicarbonate versions, with the acetate removal percentage dropping by $24-27 \%$. The difference in the high calcium variants was a less harsh $8-11 \%$ decline. This decrease in the acetate extraction represents a reduction in the targeted extractable carbonates in the high bicarbonate samples compared to their aforementioned low calcium pairs.

The majority of uranium was removed by the acetate solution (Step III) and acetic acid solution (Step IV) extractions, making a strong case for suggesting that the uranyl carbonates and silicates make up the bulk of the extracted analyte, which is consistent with predictions. In the key low bicarbonate - high calcium samples, where optimization suggested the most uranium would partition into the precipitate phase, the extraction distribution revealed that major form that uranium takes is that of uranyl silicates. This was followed closely by uranyl carbonates. In the high bicarbonate - low calcium samples, 
where supernatant analysis showed the least uranium transition into the precipitate, uranyl silicates had a similar dominant relative abundance, though the mass removed was significantly less.

\section{Electron Probe Microanalysis}

With the exception of the low bicarbonate - low calcium sample (05-00A) (Figure 24), the majority of samples, the EPMA data shows a discernable alignment of the elemental distributions of uranium and silicon (Figure 25, Figure 26 \& Figure 27). The association could signify the presence of a uranyl-silicate phase, which would be consistent with interpretations of the sequential extraction data and predictive speciation modeling which predicted the formation of species like Na-boltwoodite $\left(\mathrm{Na}\left(\mathrm{UO}_{2}\right)\left(\mathrm{SiO}_{3} \mathrm{OH}\right) \cdot \mathrm{H}_{2} \mathrm{O}\right)$ in similar systems (Lagos et al., 2016; Szecsody et al., 2012). The association could also relate to the adsorption of uranyl species to silicate sites by ion exchange or surface complexation mechanisms (McKinley, Zachara, Smith, \& Turner, 1995; Pabalan \& Turner, 1996). Similarly, the association could represent the predicted dissolution and coating of uranyl species with silicates. 


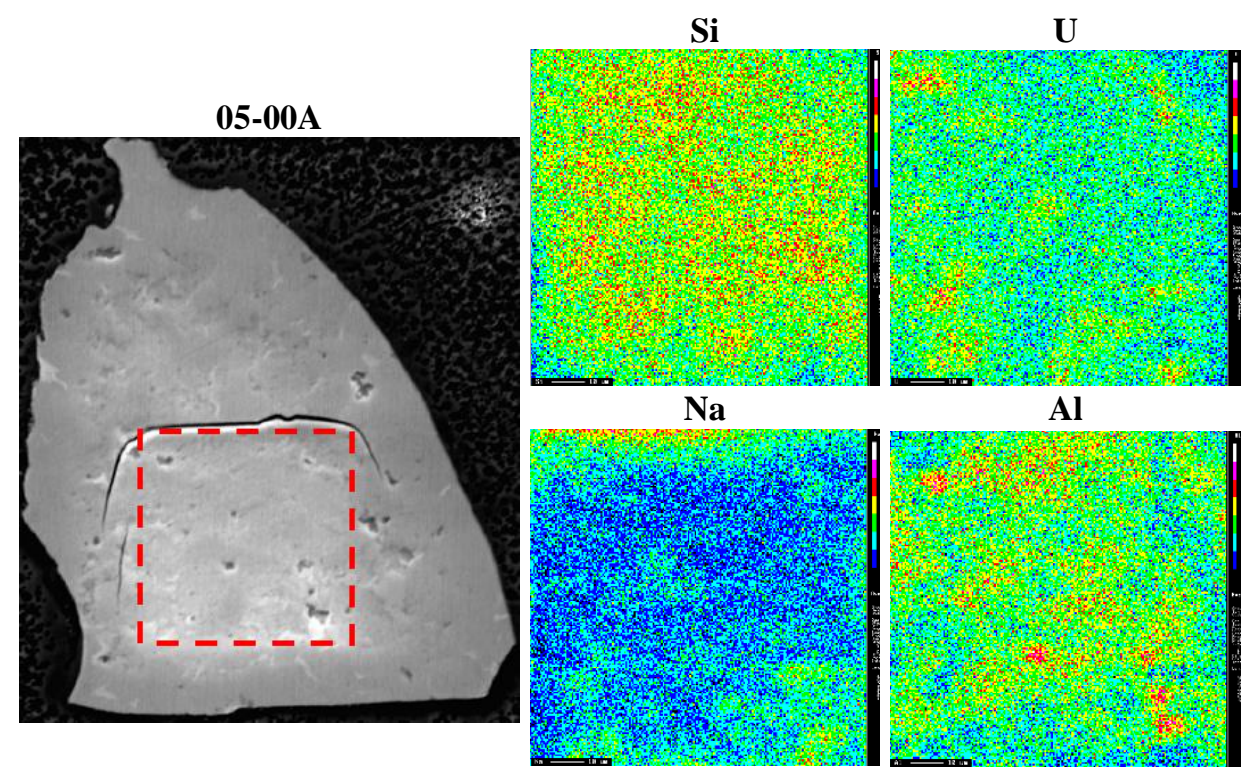

Figure 24. EPMA micrograph and corresponding elemental maps for the unrinsed, low bicarbonate $(5 \mathrm{mM})$, low calcium $(0 \mathrm{mM})$ sample

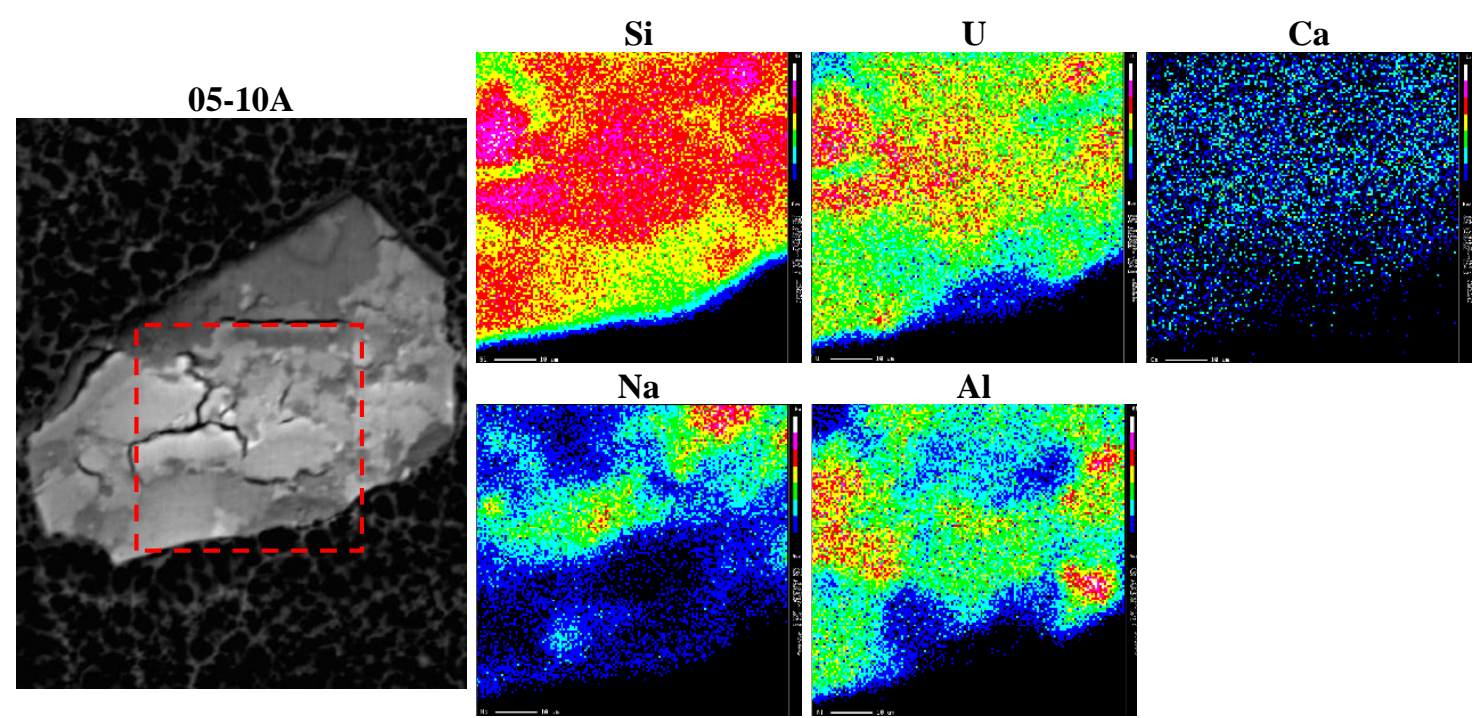

Figure 25. EPMA micrograph and corresponding elemental maps for the unrinsed, low bicarbonate $(5 \mathrm{mM}), 10 \mathrm{mM}$ calcium sample

Though aluminum distribution map seemed to show potential trends and associations with silicon and uranium, none were definitive. It could be argued that the low bicarbonate low calcium sample showed an inverse relationship between the aluminum and uranium distribution maps while there was no association between aluminum and silicon (Figure 24). In sediment samples treated with the $\mathrm{NH}_{3}$ gas, aluminosilicates would dissolve with 
$\mathrm{pH}$ elevation while other species, like aluminum hydroxide $\left(\mathrm{Al}(\mathrm{OH})_{3}\right)$, would precipitate when the system was neutralized (Szecsody et al., 2012). If $\mathrm{Al}(\mathrm{OH})_{3}$, rather than aluminosilicates, were formed in the system, it could explain the lack of any correlation between the two. Additionally, it has proposed that the carbonate species in a system could complex with surface $\mathrm{Al}(\mathrm{OH})_{3}$ particles ( $\mathrm{Su} \&$ Suarez, 1997), competitively impeding the adsorption of uranium species and thus supporting an inverse relationship. Though there is does appear to be significant association between uranium and aluminum in the high bicarbonate - low calcium sample (Figure 26), the regions with the most significant abundance of aluminum show a recession of uranium.

In the low bicarbonate - high calcium sample (05-10A), the distribution of calcium is sparse and shows no clear associations with the other elements (Figure 25). Contrastingly, the elemental distribution of the high bicarbonate - high calcium sample (50-10A) showed the calcium completely isolated; concentrated in surface structures on the sample (Figure 27). Though there is no apparent association with the other target elements, it is likely that these formations are calcium-carbonate precipitates that formed throughout the precipitate. A very small amount of uranium associated with the calcium carbonate particles which could be representative of the formation of a uranyl carbonate species, which were predicted by speciation modeling under high bicarbonate conditions (Lagos et al., 2016; Zheng, Tokunaga, \& Wan, 2003). 


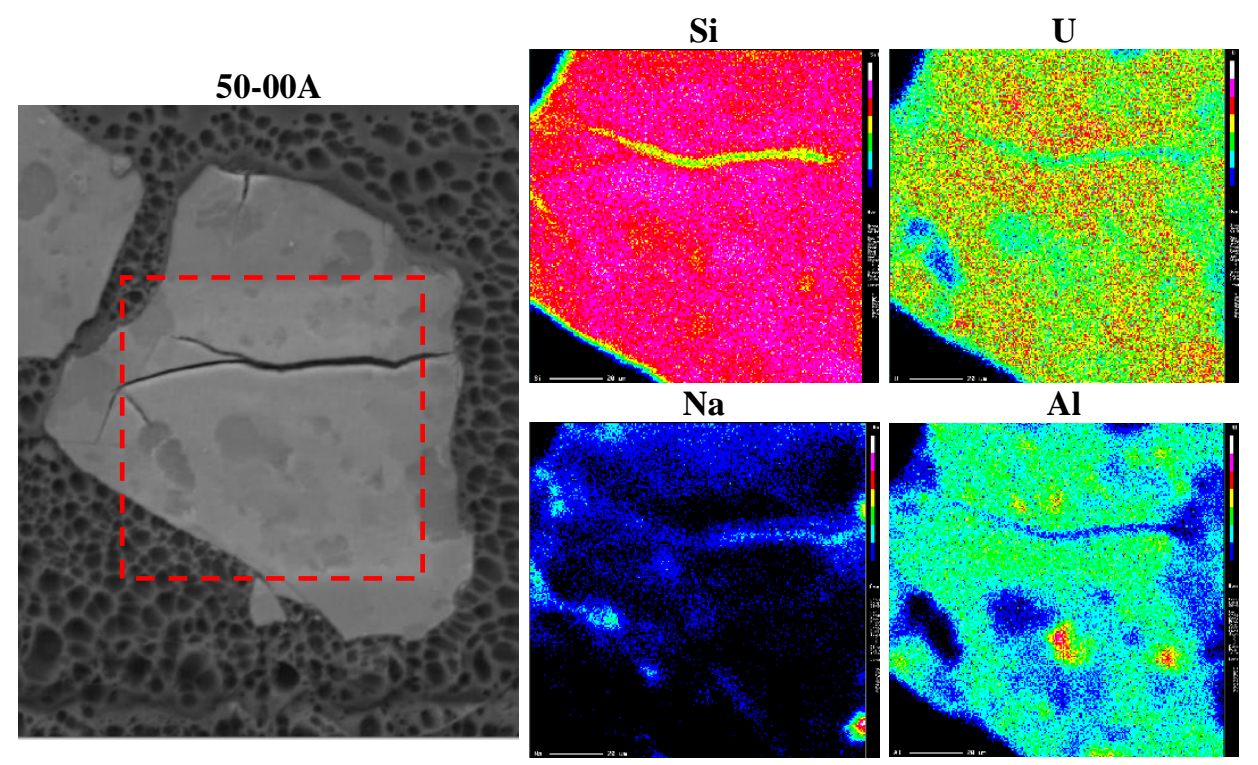

Figure 26. EPMA micrograph and corresponding elemental maps for the unrinsed, high bicarbonate $(50 \mathrm{mM})$, low calcium $(0 \mathrm{mM})$ sample

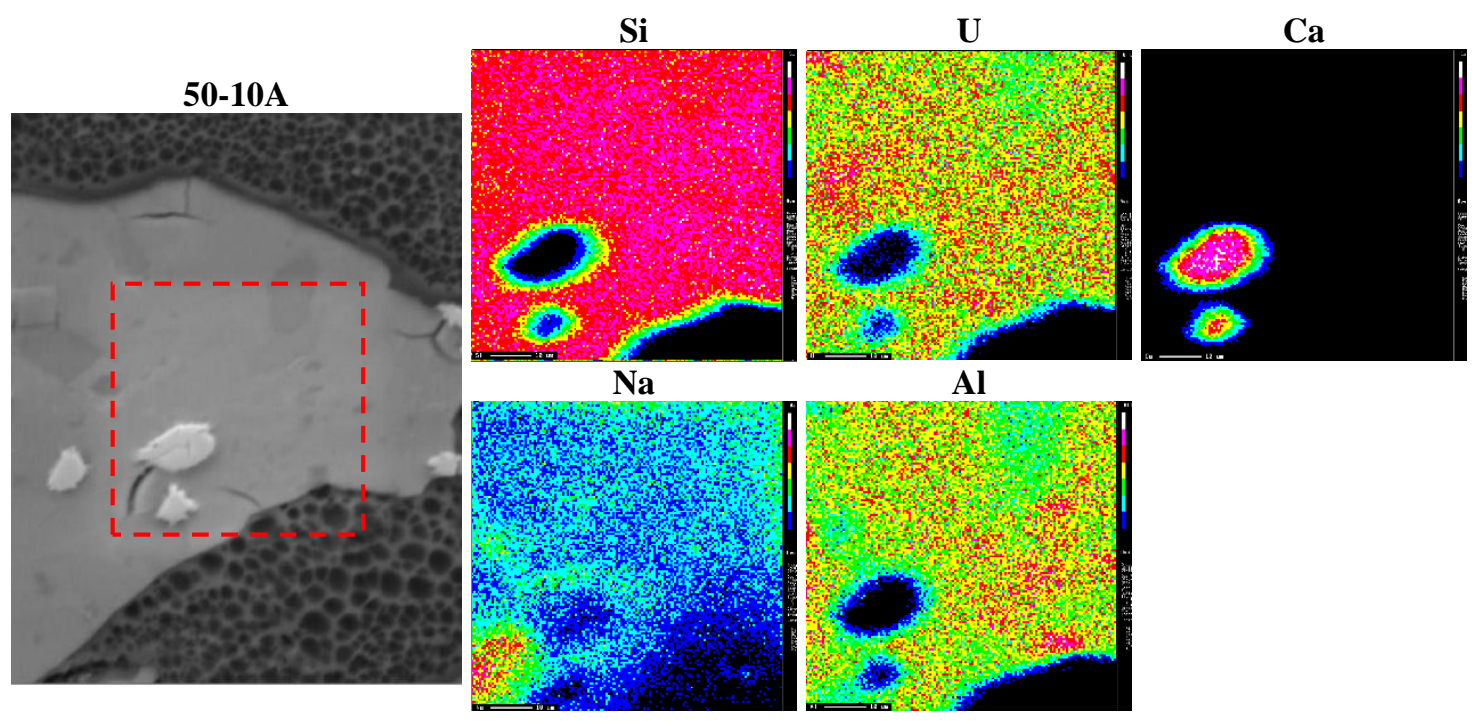

Figure 27. EPMA micrograph and corresponding elemental maps for the unrinsed, high bicarbonate $(50 \mathrm{mM}), 10 \mathrm{mM}$ calcium sample

The elemental distribution maps for sodium in the showed a tenuous association with those of uranium in all samples except for the high bicarbonate - no sodium (50-00A) analysis. The relationship could signify the presence of sodium-uranyl species like cejkaite $\left(\mathrm{Na}_{4}\left(\mathrm{UO}_{2}\right)\left(\mathrm{CO}_{3}\right)_{3}\right)$, which was identified by $\mathrm{XRD}$ as a potential identity for the uranium rich sites observed in SEM-EDS analysis. Working against this idea is the fact that the 
uranium-laden species that were thought to be cejkaite were only observed in the high bicarbonate samples while the proposed trend is more apparent in the low bicarbonate samples. 


\section{Conclusion and Recommendations}

The ammonia gas injection remediation method was replicated using synthetic pore water solutions designed to emulate conditions relevant to the Hanford 200 Area vadose zone. The initial analysis of precipitates showed significant uranium-rich crystalline species which were not observed in samples prepared with procedures modified to limit the precipitation of otherwise soluble uranium species. Coupled with the results of SEM-EDS and XRD analysis, the findings strongly suggest that the observed uranium rich phases were likely to have been uranyl-carbonates (i.e.: cejkaite), possibly precipitated during the evaporation of the residual supernatant during the drying step.

Analysis of the filtered supernatants of the samples prepared with the modified preparation procedure showed a distinct positive correlation between the increasing concentrations of bicarbonate in the initial solutions and the concentration of uranium in the filtrate. The correlation is believed to be associated with the formation of aqueous uranyl-carbonate species that promote the retention of uranium in the aqueous phase. Similarly, increased carbonate concentrations are tied to an increase in uranium mobility in the Hanford vadose zone (Zachara et al., 2007). The experimental results showed a simultaneous decrease in the uranium in the supernatant with increasing calcium content that was likely indicative of the formation of more stable calcium-uranyl-carbonate phases being removed from the solution.

There is agreement between the analysis of the sample supernatants and the elemental analysis of precipitates in that associations between carbon and uranium abundance, determined to be indicative of the formation of uranyl-carbonate species, was primarily 
observed in higher bicarbonate samples. The low-bicarbonate samples, which were thought to be most likely to precipitate uranium species because of the relatively low retention of uranium in their supernatants, presented no regions that reached atomic percentages of $1 \%$. Despite this, both SEM-EDS analysis and EPMA analysis showed reasonable associations between uranium and silicon which were proposed to represent either the formation of uranyl-silicates or the coating of uranium species with precipitated silicates. Elemental mapping of samples via electron probe microanalysis did not offer strong associations between calcium and uranium but did show that calcium carbonate precipitated at high bicarbonate concentrations, likely reducing calcium availability for formation of the more stable calcium-uranyl-carbonates and increasing the mobility relative to the low bicarbonate - low calcium samples.

The results of sequential extraction experiments provided further support for the classification of the uranium-bearing species being largely made up of uranyl-carbonates and uranyl-silicates. The bulk of the uranium in these samples were extracted in the acetate (Step III) and acetic acid (Step IV) extractions which targeted carbonates and silicates, respectively. The association supports predictions that the major precipitate formed in similar systems would be Na-boltwoodite, a uranyl-silicate (Szecsody et al., 2010; Szecsody et al., 2012). The data also revealed that the low bicarbonate samples produced more uranium in their precipitates than their high bicarbonate counterparts. The carbonate extraction solution (Step II), targeting adsorbed species, in particular was significantly larger in the low bicarbonate samples than their high bicarbonate counterparts supporting the idea that the additional carbonates in solution resulted in more mobility by reducing adsorption of uranium species. Similarly, each of the calcium-containing samples had more 
adsorbed uranium species removed than their calcium-free counterparts, supporting the idea of the formation of more stable, less mobile calcium-uranyl-carbonate species.

The results of the characterization of the uranium-bearing products collectively agree that both uranyl-carbonates and uranyl silicates were most likely formed in the sample precipitates. It is apparent that carbonate content was important to the speciation of uranium, with increased bicarbonate concentrations resulting in increased uranium mobility. The addition of calcium to the system appeared to favor the formation more stable species and reduce the mobility of uranium in the system. In future analysis, the characterization study would include variant silicon concentrations to observe if trend information would reveal more about the formation of the predicted uranyl silicates. Additionally, the sequential extraction procedure could benefit from a $6^{\text {th }}$ extraction step for the complete digestion of the sample to ensure that any remaining uranium was accounted for.

Research involving the use of a radioactive actinide like uranium will often call for specialized facilities and equipment familiar with, if not dedicated to, working with this class of hazardous material. During the course of my investigation, access to multiple facilities with desired analytical capabilities was denied either because of the nature of the samples or the non-selection of a collaboration proposal. Structural analysis using x-ray diffraction was completed with initial samples but limited access to a facility amenable to working with a uranium-containing solid prevented the diffraction analysis of the final samples. As such, it wasn't possible to form a positive identification of any specific uranylcarbonate or uranyl-silicate species. Future work would include re-applying for access to 
the Environmental Molecular Sciences Laboratory (EMSL) which has a dedicated rad-lab and writing a proposal for synchrotron beam time at Argonne National Laboratory for high energy analysis. There, $x$-ray spectroscopy and single-crystal x-ray diffraction for sample precipitates could be used to reach a conclusive identification of the uranium phases being formed. 


\section{References}

Ariza, J. G., Giraldez, I., Sanchez-Rodas, D., \& Morales, E. (2000). Metal sequential extraction procedure optimized for heavily polluted and iron oxide rich sediments. Analytica Chimica Acta, 414(1-2), 151-164.

Cantrell, K. J., Zachara, J. M., Dresel, P. E., Krupka, K. M., \& Serne, R. J. (2007). Geochemical processes data package for the vadose zone in the single-shell tank waste management areas at the hanford site. ( No. PNNL-16663).Pacific Northwest National Laboratory.

Galán, E., Gómez-Ariza, J., González, I., Fernández-Caliani, J., Morales, E., \& Giráldez, I. (2003). Heavy metal partitioning in river sediments severely polluted by acid mine drainage in the iberian pyrite belt. Applied Geochemistry, 18(3), 409-421.

Gee, G. W., Oostrom, M., Freshley, M. D., Rockhold, M. L., \& Zachara, J. M. (2007). Hanford site vadose zone studies: An overview. Vadose Zone Journal, 6, 899.

Gephart, R. E. (2003). A short history of hanford waste generation, storage, and release . ().Pacific Northwest National Laboratory.

Gephart, R. E. (2010). A short history of waste management at the hanford site. Physics and Chemistry of the Earth, Parts A/B/C, 35(6-8), 298-306. doi:http://dx.doi.org.ezproxy.fiu.edu/10.1016/j.pce.2010.03.032

Gerber, M. S. (1992). Legend and legacy: Fifty years of defense production at the hanford site. ( No. WHC-MR-0293).Westinghouse Hanford Company. Retrieved from http://www.osti.gov/scitech//servlets/purl/10144167-kRWeQd/webviewable/

Hartman, M. J., \& Dresel, P. E. (1998). Hanford groundwater monitoring for fiscal year 1997. ( No. PNNL-11793).

Hughes, M. C., Douglas, L. M., \& Marske, S. G. (1994). Accelerated decontamination and decommissioning at the hanford site

Iler, R. K. (1979). The chemistry of silica: Solubility, polimerization, colloid and surface properties, and biochemistry Wiley Interscience.

Kohler, M., Curtis, G. P., Meece, D. E., \& Davis, J. A. (2004). Methods for estimating adsorbed uranium (VI) and distribution coefficients of contaminated sediments. Environmental Science \& Technology, 38(1), 240-247.

Lagos, L., Katsenovich, Y., Gudavalli, R., Cardona, C., Lapierre, R., Sepulveda, P., . . . Pino, C. (2014). Year-end technical report - rapid deployment of engineered 
solutions for environmental problems at hanford. ().Florida International University's Applied Research Center.

Lagos, L., Katsenovich, Y., Gudavalli, R., Cardona, C., Moreno, C., \& Sepulveda, P. (2012). Year-end technical report - rapid deployment of engineered solutions for environmental problems at hanford. ().Florida International University's Applied Research Center.

Lagos, L., Katsenovich, Y., Gudavalli, R., Cardona, C., Musaramthota, V., Sepulveda, P., ... Padilla, V. (2013). Year-end technical report - rapid deployment of engineered solutions for environmental problems at hanford. ().Florida International University's Applied Research Center.

Lagos, L., Katsenovich, Y., Gudavalli, R., Lawrence, A., Garcia, R., Setegn, S., . . . Abarca, A. (2016). Year-end technical report - rapid deployment of engineered solutions for environmental problems at hanford. ().Florida International University's Applied Research Center.

Liu, C., Jeon, B., Zachara, J. M., Wang, Z., Dohnalkova, A., \& Fredrickson, J. K. (2006). Kinetics of microbial reduction of solid phase U(VI). Environmental Science \& Technology, 40(20), 6290-6296. doi:10.1021/es0608601

Liu, C., Zachara, J. M., Zhong, L., Heald, S. M., Wang, Z., Jeon, B., \& Fredrickson, J. K. (2009). Microbial reduction of intragrain U(VI) in contaminated sediment. Environmental Science \& Technology, 43(13), 4928-4933. doi:10.1021/es8029208

McKinley, J. P., Zachara, J. M., Smith, S. C., \& Turner, G. D. (1995). The influence of uranyl hydrolysis and multiple site-binding reactions on adsorption of $U$ (VI) to montmorillonite.

Pabalan, R. T., \& Turner, D. R. (1996). Uranium (6 ) sorption on montmorillonite: Experimental and surface complexation modeling study. Aquatic Geochemistry, 2(3), 203-226.

Rauret, G., Lopez-Sanchez, J., Sahuquillo, A., Rubio, R., Davidson, C., Ure, A., \& Quevauviller, P. (1999). Improvement of the BCR three step sequential extraction procedure prior to the certification of new sediment and soil reference materials. Journal of Environmental Monitoring, 1(1), 57-61.

Schultz, M. K., Burnett, W. C., \& Inn, K. G. (1998). Evaluation of a sequential extraction method for determining actinide fractionation in soils and sediments. Journal of Environmental Radioactivity, 40(2), 155-174.

Serne, R. J., Last, G. V., Gee, G. W., Schaef, H. T., Lanigan, D. C., Lindenmeier, C. W., . . . Vickerman, T. S. (2008). Characterization of vadose zone sediment: Borehole 
299-E33-45 near BX-102 in the B-BX-BY waste management area. ( No. PNNL14083 rev.1).Pacific Northwest National Laboratory.

Smith, S. C., \& Szecsody, J. E. (2011). Influence of contact time on the extraction of 233uranyl spike and contaminant uranium from hanford site sediment. Radiochimica Acta International Journal for Chemical Aspects of Nuclear Science and Technology, 99(11), 693-704.

Su, C., \& Suarez, D. L. (1997). In situ infrared speciation of adsorbed carbonate on aluminum and iron oxides.

Szecsody, J. E., Truex, M. J., Zhong, L., Williams, M. D., Resch, C. T., \& McKinley, J. P. (2010). Remediation of uranium in the hanford vadose zone using gas-transported reactants: Laboratory scale experiments in support of the deep vadose zone treatability test plan for the hanford central plateau. ( No. PNNL-18879).Pacific Northwest National Laboratory (PNNL), Richland, WA (US).

Szecsody, J. E., Truex, M. J., Zhong, L., Johnson, T. C., Qafoku, N. P., Williams, M. D., ... Faurie, D. K. (2012). Geochemical and geophysical changes during ammonia gas treatment of vadose zone sediments for uranium remediation. Vadose Zone Journal, 11(4) doi:10.2136/vzj2011.0158

Szecsody, J. E., Truex, M. J., Zhong, L., Qafoku, N. P., Williams, M. D., McKinley, J. P., ... Phillips, J. L. (2010). Remediation of uranium in the hanford vadose zone using ammonia gas. ( No. PNNL-20004).Pacific Northwest National Laboratory.

Szecsody, J. E., Truex, M. J., Zhong, L., Williams, M. D., Resch, C. T., \& McKinley, J. P. (2010). Remediation of uranium in the hanford vadose zone using gas-transported reactants. ( No. PNNL-18879).Pacific Northwest National Laboratory.

Tessier, A., Campbell, P. G., \& Bisson, M. (1979). Sequential extraction procedure for the speciation of particulate trace metals. Analytical Chemistry, 51(7), 844-851.

U.S. Department of Energy. (2018). Hanford. Retrieved from www.hanford.gov

U.S. Department of Energy - Richland Operations Office. (2008). Deep vadose zone treatability test plan for the hanford central plateau. ( No. DOE/RL-200756).United States Department of Energy.

Zachara, J. M., Brown, C. F., Christensen, J. N., Davis, J. A., Dresel, P. E., Liu, C., . . Um, W. (2007). A site wide perspective on uranium geochemistry at the hanford site. ( No. PNNL-17031). Retrieved from http://www.osti.gov/scitech//servlets/purl/920206/ 
Zheng, Z., Tokunaga, T. K., \& Wan, J. (2003). Influence of calcium carbonate on U (VI) sorption to soils. Environmental Science \& Technology, 37(24), 5603-5608.

Zhong, L., Szecsody, J. E., Truex, M. J., Williams, M. D., \& Liu, Y. (2015). Ammonia gas transport and reactions in unsaturated sediments. Journal of Hazardous Materials, 289, 118. doi:10.1016/j.jhazmat.2015.02.025 